\title{
EFFECTS OF BIAXIAL STRETCH ON ARTERIOLAR FUNCTION IN VITRO
}

\author{
A Thesis \\ by \\ HONG GUO \\ Submitted to the Office of Graduate Studies of \\ Texas A\&M University \\ in partial fulfillment of the requirements for the degree of \\ MASTER OF SCIENCE
}

August 2006

Major Subject: Biomedical Engineering 


\title{
EFFECTS OF BIAXIAL STRETCH ON ARTERIOLAR FUNCTION IN VITRO
}

\author{
A Thesis \\ by \\ HONG GUO
}

\author{
Submitted to the Office of Graduate Studies of \\ Texas A\&M University \\ in partial fulfillment of the requirements for the degree of \\ MASTER OF SCIENCE
}

\begin{abstract}
Approved by:
Chair of Committee, Jay D. Humphrey

Committee Members, Wonmuk Hwang

Alvin T. Yeh

David C. Zawieja

Head of Department, Gerard L. Coté
\end{abstract}

August 2006

Major Subject: Biomedical Engineering 


\author{
ABSTRACT \\ Effects of Biaxial Stretch on Arteriolar Function In Vitro. (August 2006) \\ Hong Guo, B.S., Fudan University, China \\ Chair of Advisory Committee: Dr. Jay D. Humphrey
}

Mounting evidence suggests that the normal biomechanical state of arteries may include a nearly equibiaxial intramural stress, and that arteries tend to undergo rapid and dramatic remodeling when perturbed from this normal state. Technical developments in the early 1980s and late 1990s enabled in vitro and ex vivo studies, respectively, of isolated perfused microvessels, and it is clear that they share many similarities in behavior with arteries. To date, however, there has been no systematic study of the effects of biaxial loading on the biomechanical behavior of arterioles. In this project, we describe a modification to a prior in vitro arteriole test system that allowed us to investigate the role of altered axial stretch on the passive, myogenic, and fully contracted biaxial behavior of isolated rat cremaster arterioles. We show that axial stretches from $85 \%$ to $110 \%$ of normal values induce modest changes in the measured circumferential and axial stress-stretch behavior and similarly in traditional measures of distensibility and myogenic index. Nevertheless, altered axial stretch has a dramatic affect on the biaxial state of stress and it appears that near equibiaxial stress occur at axial stretches larger than those used previously. Whereas this finding will not affect prior estimates of material and functional behavior, it may have important implications for the design of long-term ex vivo and in vivo studies wherein vessel growth and remodeling are critical. 
To my parents,

Yonghua Guo and Meiying Luo 


\section{ACKNOWLEDGMENTS}

I am extremely grateful to my supervisors, Dr. Jay Humphrey and Dr. Michael Davis, for their support and guidance throughout the course of this research. Their passion and dedication to biomedical research will always be inspiring to me. 


\section{TABLE OF CONTENTS}

Page

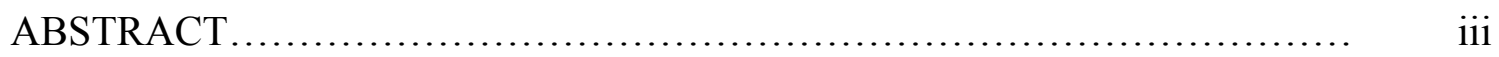

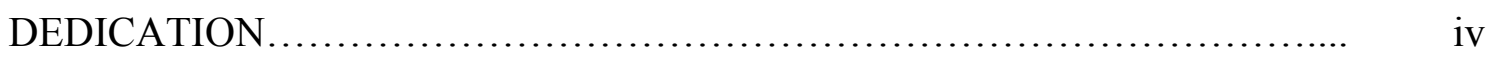

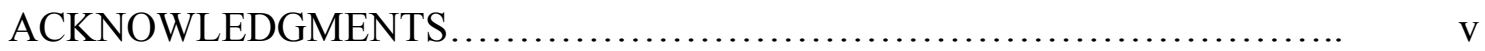

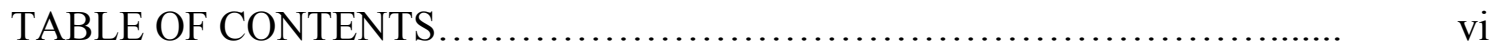

LIST OF TABLES..................................................... viii

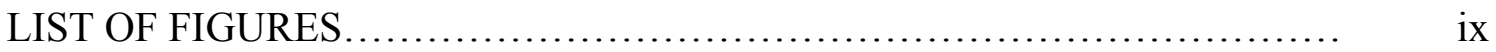

\section{CHAPTER}

I INTRODUCTION.............................................. 1

II $\quad$ BACKGROUND................................................ 3

Introduction.......................................... 3

Arteriolar composition.................................. 4

Passive mechanical behaviors........................... 5

Mechanics of myogenic response...................... 12

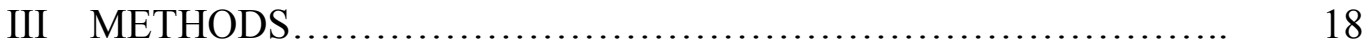

Preparation......................................... 18

Experimental protocols............................... $\quad 20$

Useful metrics....................................... 22

Data analysis..................................... 24

IV RESULTS............................................. 25

Morphological data..................................... 25

Diameter vs. pressure and NE........................ 27

Circumferential stress vs. circumferential stretch ratio........ 29

Axial stress vs. axial stretch ratio....................... 32

Stress ratio vs. pressure and NE.......................... 34 
CHAPTER Page

Incremental distensibility, myogenic index, and constriction ratio......................................... 35

V DISCUSSION......................................... 38

VI CONCLUSIONS.............................................. 46

REFERENCES ........................................................... 48






\section{LIST OF TABLES}

TABLE Page

1 Volume density of normal rat cerebral pial arterioles..................... 5

2 Average axial stretch ratios for low, moderate, and high stretch groups........ 25

3 Morphological characteristics of arterioles in unloaded and pressurized

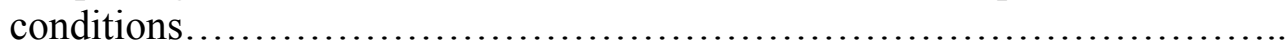




\section{LIST OF FIGURES}

FIGURE Page

1 Experimental setup of the arteriolar biaxial stretch test and corresponding

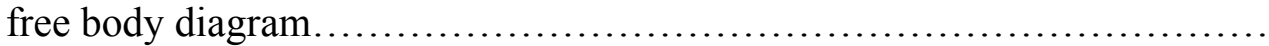

2 Change in diameter as a function of intraluminal pressure or NE concentration at low, moderate, or high axial stretch.

3 Relationship between circumferential stress $\sigma_{\theta}$ and circumferential stretch ratio $\lambda_{\theta}$ for passive, myogenic, and NE responses at low, moderate, or high axial stretch

4 Relationship between axial stress $\sigma_{z}$ and axial stretch ratio $\lambda_{z}$ for passive, myogenic and NE responses..............................................

5 Change of stress ratio $\sigma_{\theta} / \sigma_{\mathrm{z}}$ as a function of intraluminal pressure or $\mathrm{NE}$ at low, moderate, and high levels of axial stretch....

6 Incremental distensibility and myogenic index at low, moderate and high axial stretch

7 Change of constriction ratio as a function of intraluminal pressure or $\mathrm{NE}$ at low, moderate and high axial stretch 


\section{CHAPTER I}

\section{INTRODUCTION}

Smooth muscle constitutes two-thirds of arteriolar wall volume $(7,9)$; this thick muscular wall allows arterioles to actively constrict and relax in response to remote and local stimuli, and thereby to change local resistance and blood flow $(19,21)$. In this way, arterioles are the major vascular segment controlling peripheral resistance. Among local vasoactive control mechanisms, the pressure-induced, myogenic response plays a primary role $(42,68)$.

The effects of smooth muscle activation on the wall mechanics of small arteries has been a topic of extensive investigation since the 1970s $(16,17)$. Improved methods for in vitro isolation, cannulation, and study of microvessels $(25,69)$ have enabled detailed functional studies, e.g., on the myogenic response and smooth muscle activation in health and disease $(52,53)$. It is recognized that cellular mechanisms underlying the

myogenic response, such as activation of voltage-gated channels and $\mathrm{Ca}^{2+}$ influx, are initiated by alterations in the mechanical state of the vascular smooth muscle (60). Acknowledged as a process of mechanotransduction (21), the myogenic response has been hypothesized to reflect intrinsic mechanisms that maintain wall tension and/or circumferential stress near a homeostatic value $(11,19,41,48)$.

This thesis follows the style of American Journal of Physiology - Heart and Circulatory Physiology. 
Despite a number of important physiological and pharmacological studies of isolated, pressurized arterioles, the biomechanical properties of arterioles have not been fully characterized. For example, axial force is known to significantly alter both the response of smooth muscle (76) and the remodeling of arteries (32), but other than anecdotal reports its influence on arteriolar responsiveness has not been measured or quantitated systematically.

The present study sought to delineate passive and active wall mechanics of arterioles under in-plane biaxial (circumferential and axial) loading with reference to an unloaded configuration. We tested the hypothesis that there is an optimal mechanical state in vitro for the arteriolar myogenic response and for agonist-induced vasoconstriction. We also hypothesized that the dominance of smooth muscle in the arteriolar wall and its relatively high responsiveness distinguish its wall mechanics from large vessels and present additional complications for mathematical modeling. Thus, a secondary objective was to collect experimental data that support a nonlinear biomechanics approach to modeling arteriolar behavior similar to that which has been used to study the mechanics of large arteries $(27,44)$. 


\section{CHAPTER II}

\section{BACKGROUND}

\section{Introduction}

Vascular biomechanics has been a topic of extensive investigation. Many types of mechanical methods and tools, from nonlinear elasticity $(23,24,43)$ to finite element methods $(12,50,66,71)$, have been applied to delineate the mechanical properties of vascular walls. From the early days of using the Fung exponential form to fit data from biaxial tests on blood vessels and to calculate stress and strain distributions, to current approaches of using atomic force microscope (AFM) to measure the elasticity of single vascular smooth muscle cell (64), our knowledge of the mechanical behavior of blood vessels has advanced tremendously. Mechanical stress is now recognized as one of the most important stimuli to initiate cellular responses and thereby to regulate vascular growth and remodeling (G\&R); thus, the new terminology 'mechanobiology' has arisen. However, when we look back to what mechanics has done to advance our understanding to the properties of vasculature, it is amazing that there is a huge gap in studies of one important segment of the vascular tree: arterioles.

The mechanical behaviors of arterioles, which extend and branch out from an artery and terminate in capillaries, are less well understood when compared to the behaviors of conduit arteries. For many of the published results in physiology, the interpretations and analysis of mechanical indices are sometimes inappropriate because of an insufficient understanding of mechanics. On the other hand, a primary reason for the relative lack of 
engineering analyses on single arterioles is the technical difficulty involved in studying them. The techniques of arteriolar dissection, cannulation and perfusion, initially developed by Duling in the early 1980s, demand specialized training and much practice (25). It is difficult to get an arteriole with a comprehensive set of physiological responses, i.e., myogenic responses, endothelial-dependent responses, and pharmacological reactivity. Any mishandling in dissection or cannulation is more than enough to impair or eliminate one or more of the above arteriolar responses. Moreover, the absence of good models can be attributed to other aspects, e.g., the complexity of arteriolar reactivity, lack of support from experimental data, etc.

\section{Arteriolar composition}

Composed of a thick muscular wall, arterioles actively constrict and relax by means of myogenic, hormonal, and pharmacological mechanisms $(19,21)$. As the major vascular component that controls peripheral resistance, the arterioles are distinct from conduit arteries. The composition of arterioles is not dominated by collagen but by smooth muscle $(7,9)$, which makes up two thirds of arteriolar wall volume and determines the overall mechanical behavior. Collagen, which typically composes half of the volume of large arteries, has a negligible volume density in arteriolar composition (37).

The composition of arteriolar walls has hardly been reported with the exception of the composition of rat cerebral pial arterioles, which have been extensively studied by Baumbach and colleagues (Table 1). With wall thickness ranging from 4 to 5 microns, the arterioles in these experiments had inner diameters between 70 to 110 microns in vivo after being deactivated by EDTA $(6-8,37)$. Using point counting stereology, it was 
found that the composition of rat pial arteriolar walls can be altered by many factors, including hypertension, age, etc $(5-9,36,37)$. Although aging and hypertension can alter arteriolar wall composition in a statistical sense, the degree of change is low, e.g.., the volume density of smooth muscle did not exceed $\pm 10 \%$ of its normal/control value $(5,8$, $9,36,37)$. These studies focused largely on reporting arteriolar wall composition and mechanical behaviors; nevertheless, the physiological and cellular mechanisms underlying arteriolar G\&R have not been explored. Human subcutaneous resistance arteries have been shown to have similar wall composition to rat cerebral pial arterioles (46), which may imply that the resistance arteries and large arterioles share some common characteristics in mechanical properties. For resistance arteries, on the other hand, the compositional, morphological and functional changes of the vessel wall under G\&R have been reported more extensively $(29,39,63,65,70,72)$.

Table 1. Volume density of normal rat cerebral pial arterioles

\begin{tabular}{|l|l|l|l|l|l|l|}
\hline Volume density (\%) & $\begin{array}{l}\text { Baumbach } \\
(7)\end{array}$ & $\begin{array}{l}\text { Baumbach } \\
(6)\end{array}$ & $\begin{array}{l}\text { Hajdu } \\
(36)\end{array}$ & $\begin{array}{l}\text { Hajdu } \\
(37)\end{array}$ & $\begin{array}{l}\text { Baumbach } \\
(8)\end{array}$ & $\begin{array}{l}\text { Baumbach } \\
(5)\end{array}$ \\
\hline Smooth muscle & $66.9 \pm 0.8$ & $65 \pm 1.0$ & $66 \pm 1.7$ & $66.0 \pm 0.76$ & $63 \pm 1$ & $66.0 \pm 0.2$ \\
Elastin & $7.3 \pm 0.4$ & $6 \pm 0.4$ & $8 \pm 0.3$ & $5.7 \pm 0.36$ & $8 \pm 0.4$ & $6.0 \pm 0.6$ \\
Collagen & $0.7 \pm 0.08$ & $0.5 \pm 0.04$ & $0.9 \pm 0.11$ & $0.2 \pm 0.02$ & $0.7 \pm 0.09$ & $0.3 \pm 0.1$ \\
Basement membrane & $9.9 \pm 0.4$ & $11 \pm 0.4$ & $12 \pm 0.6$ & $13.0 \pm 0.60$ & $13 \pm 0.5$ & $12.0 \pm 1.1$ \\
Endothelium & $15.2 \pm 0.9$ & $18 \pm 0.8$ & $14 \pm 0.9$ & $15.1 \pm 1.07$ & $16 \pm 0.7$ & $16.0 \pm 0.8$ \\
\hline
\end{tabular}

Values are means $\pm \mathrm{SE}$.

\section{Passive mechanical behaviors}

The pressure-diameter plot is the most common way to depict the passive mechanical behavior of microvessels. Circumferential wall tension and incremental distensibility can 
be calculated from recorded vessel diameter and luminal pressure. Using an in vivo method, passive arteriolar behaviors have been reported for normal, aged, and hypertensive rat cerebral pial arterioles after deactivation by suffusion of CSF containing EDTA $(5-9,36,37)$. Measurements of pressure-diameter relationships are followed by calculations of circumferential stress and incremental distensibility. As the studies are conducted in vivo, the pressure-diameter responses do not reflect the intrinsic properties of the arteriolar wall well since the influence of peripheral tissue is not excluded. Passive arteriolar behaviors have also been studied in vitro for hamster check pouch (19, $20,34,48)$, rat gracilis anticus muscle (73), rat mesentery (74), pig subepicardium and subendocardium (54), and human coronary (61) arterioles. In these same studies, arteriolar myogenic responses are also investigated. The passive pressure-diameter responses actually serve as reference for the active responses. For resistance arteries with diameters of 200 to 300 microns, passive mechanical behaviors have been reported for isolated, pressurized vessels from rat mesentery $(55,56,65)$, rat gastrocnemius and soleus muscle (62), rat coronary (75), and human subcutaneous tissue (46) to study the effects of age and hypertension. In most cases, the results are followed by calculations of wall stress and incremental distensibility. Recently, the pressure-diameter curve of rat cremaster arterioles has been examined in the studies of arteriolar growth and remodeling (2-4), however, other mechanical parameters like stress and distensibility were not calculated.

Though a pressure-diameter curve retains its simplicity and beauty, the information provided is incomplete. Studies of the mechanical properties of microvessels will be 
more useful if the responses reveal the distinctive properties of the vessels and facilitate mechanical modeling. The stress-strain relationship, intrinsically similar to the pressurediameter relationship, reflects the uniqueness of material properties of the vessel wall and is likely to be more useful for biomechanical modeling.

Incremental distensibility is another mechanical parameter that has been used to delineate passive mechanical properties of microvessels. Physically interpreted as the change of luminal diameter in response to a change of luminal pressure, incremental distensibility can be plotted from inflation tests as only pressure and diameter are required for computing the parameter. Unlike the calculation of strain, no unique reference configuration is needed for computing incremental distensibility. This mechanical parameter has been reported from in vivo studies of rat pial cerebral arterioles $(5,7,8)$ as well as experiments performed in vitro for rat mesentery resistance arteries $(55,56)$. As incremental distensibility is computed by normalization of diameter, the effect of vessel size is eliminated. As long as the pressure-diameter curves parallel each other or overlap, the curves of incremental distensibility will overlap. Although incremental distensibility describes the structural properties of microvessel walls fairly well, its lack of physical meaning and inability of being adapted into mechanical modeling limit its utility.

Wall stresses have been extensively studied in vivo for rat pial arterioles $(5-8,37)$. Worthy of mention, the reference diameter used for computing strain in those studies is not measured at an intraluminal pressure near zero but extrapolated. It is interesting to see how the stress-strain curves are interpreted in the studies. As an example, the stress- 
strain curve has a greater slope, i.e., the elastic modulus, for pial arterioles in rat cerebrum than in the brain stem (7). Elastic modulus computed from a stress-strain curve is a different mechanical parameter from incremental distensibility. The lower elastic modulus of arterioles in brain stem compared to cerebrum is suggested to support the conclusion that arterioles in the brain stem have a higher distensibility, which is demonstrated by a separate plot of incremental distensibility and is further illustrated by the ratio of nondistensible components to distensible components in the arteriolar wall.

Few stress-strain relationships have been reported for isolated and cannulated arterioles. As an exception, a passive stress-strain (stress-stretch) relationship was reported for isolated skeletal muscle arterioles for the study of the effects of aging on arteriolar mechanical properties (62). The authors acknowledge that the strains computed from measuring reference diameters at zero pressure seem to be too high when the results are compared to other studies, and suggest that measurement of diameter for a collapsed arteriolar lumen at zero pressure induces errors into the strain calculation. It is interesting that the authors show two different stress-strain curves that yield contradictory conclusions. When the reference diameter is chosen at zero pressure, the arterioles from young soleus are more elastic than those from old soleus. But the use of a reference diameter set at $10 \mathrm{cmH}_{2} \mathrm{O}$ pressure leads to the conclusion that arterioles from both young and old soleus have a similar elastic modulus, as the stress-strain curves overlap. Indeed, as the reference diameter chosen at a pressure of $10 \mathrm{cmH}_{2} \mathrm{O}$ is larger, the range of strain was consequently reduced, which induces overlapping of the two stress-strain curves. 
Both passive and active stress-strain (stress-length) relationships have been studied for isolated arterioles to explore the segmental difference and regulatory mechanism of myogenic response (20). However, the reference diameter for calculating the normalized diameter has been chosen as the maximum diameter rather than the diameter in the unloaded configuration. Consequently, it makes the comparison to other studies difficult.

Segmental differences in mechanical properties have also been studied using an in vitro approach for coronary resistance arteries of rat (75). Stress- and elastic moduluspressure curves have been reported. There is no reference diameter selected for the study, thus the strains were not reported, which makes it impossible to compare the studies to other results of stress-strain relationships.

Stress-strain relationships have been studied for resistance arteries. Studies of mechanical properties of mesentery resistance arteries reveal the effect of age and hypertension on the stress-strain relationship $(55,56,65)$. Nevertheless, the strains can be difficult to interpret as the reference diameters were chosen to be the arterial diameters at different luminal pressures, e.g., at one (56), three (65) or five (55) $\mathrm{mmHg}$.

Unlike in vivo experimental studies that physically prohibit complete unloading of arteriolar wall stress (7), in vitro studies of microvessel mechanical properties are required to choose a reference configuration for calculation of strain or stretch. However, problems arise from the inconsistent reference points for computing strains and stress. The reference diameter can be picked at zero pressure, and accordingly the collapsed lumen induces measurement errors (62), whereas other in vitro studies pick reference configurations at different intraluminal pressures close to zero $(55,56,65)$. It is shown 
that the stress-strain curves computed based on reference diameters selected at non-zero pressure and zero pressure can lead to contradictory results (62). In this way, inappropriate selection of reference diameter may lead to ill-presented stress-strain curves, and hence elastic modulus, though curves of incremental distensibility are not affected as they are independent of reference diameters. Therefore, the reference diameter should be recorded at a pressure close to zero so that the reference diameter retains the uniqueness as the unloaded diameter and meanwhile maintains the mechanical meaning.

Even if a reliable reference luminal diameter is picked for strain calculations, the unloaded configuration selected in the studies for microvessels usually only establishes a pseudo load-free state. Circumferentially, the stress in the vessel wall is eliminated by lowering luminal pressure to zero or to a value near zero $(7,55,56,62)$. However, the axial stress still remains in the vessel wall. Thus, the stress-strain calculation is not established on a true unloaded configuration.

There has been extensive discussion of the unloaded configuration for large vessels. It is agreed that an unloaded configuration is established biaxially by reducing axial vessel length and zeroing luminal pressure simultaneously $(27,28,44)$. For arteriolar studies, particularly in vitro studies in which axial stretch and loading are not properly adjusted, questions will arise about the effects of axial loading on passive mechanical parameters like distensibility and elastic modulus. At zero or near-zero intraluminal pressure, luminal diameter will increase as the arteriolar axial loading is reduced by shortening the axial length from a straight shape to a nearly-bent configuration. Therefore, even if the 
luminal pressure is decreased to zero for recording reference diameter, the calculated strain may still produce different stress-strain curves if the unloaded configuration in axial direction is not considered.

As noted, different selections of unloaded reference diameters may affect stress-strain curves, and hence elastic modulus, but not incremental distensibility. However, both incremental distensibility and stress-strain curves will be changed if the selection of the axial configuration is altered. When the method of microvessel cannulation and perfusion originally developed by Duling (25) is adopted for testing, the microvessels are stretched axially to a reference length determined by experience, followed by functional or mechanical studies $(2-4,19,20,40,41,51,52,55,56,79)$. However, there is no rigorous standard for selecting the axial length for the tests. Even if microvessels are from the same tissue of same aged animals and of similar size, different arteriolar axial configurations could yield differences in elastic modulus and incremental distensibility. The pressure-diameter relationship and stress-strain curve can be speculated to change if there is a slight alteration in the axial stretch. Hence studies of mechanical properties for microvessels of different sizes, branching orders, and ages are less convincing without considering the effects of axial configuration.

In addition, the axial configuration is intrinsically associated with the growth index, i.e., a parameter using increment of wall cross-sectional area to characterize microvessel growth (55). Cross-sectional area is a function of the level of axial stretch because wall volume is conserved in response to transient loads. Thus, vessels of same size tested at different axial stretch levels will demonstrate differences in wall cross-sectional area. 
Therefore, the growth index needs to be studied under the condition that the vessels are axially stretched to the same degree. However, the authors have not considered the possible influence of axial configuration.

In summary, inappropriate selection of reference configuration and a lack of consideration in the effects of axial configuration may comprise prior studies of the mechanical properties of microvessels and the associated medically important indices, e.g., incremental distensibility and elastic modulus. Therefore, an insightful study of the axial mechanical properties and the consequent effects on microvessel functions is essential.

Mechanics of myogenic response

Although studies for passive mechanical behaviors may uncover special patterns of arteriolar mechanical behaviors, it is even more important to study and characterize unique arteriolar responses such as the myogenic response. First discovered by Bayliss, who deduced from experiments that a large part of vascular tone relies purely on intravascular pressure (10), vascular myogenic response has been quantified by different approaches. Under the isometric approach, vascular rings or strips are uniaxially stretched to test smooth muscle activation and the secondary force. On the other hand, cannulated and pressurized vessels are tested under isobaric protocols in order to examine vasoconstriction in response to increased pressure as opposed to passive response (21). Although isometric and isobaric experiments show distinctive patterns of myogenic activation, it has been suggested that the myogenic constriction and dilation of 
pressurized vessels reflect the same mechanism demonstrated in isometric rings by stretch activation and shortening deactivation respectively.

Although there have been many studies of the myogenic response, few parameters are employed to quantitate and characterize the response. The most used parameter to delineate myogenic response, the myogenic index, has been extensively reported $(19,48$, $61,73,74)$. It is interesting to see that the formula for calculating the myogenic index is the same as the formula for computing incremental distensibility. Thus, the physical meaning of myogenic index is the change of diameter in response to a change of intraluminal pressure. Similar to incremental distensibility, the myogenic index depends solely on pressure-diameter relationships and the index is predictable based on the analysis of the slope of pressure-diameter curve. Parallel or overlapped pressurediameter curves will lead to identical curves for myogenic index. If pressure-diameter curves are not parallel or overlapped, curves of myogenic indices will be accordingly different. Similar to studies of incremental distensibility, it is not always easy to judge myogenic index by looking at the whole patterns of curves. Within the test range of luminal pressure, incremental distensibility as well as myogenic index needs to be judged one pressure point relative to another if a difference is to be exhibited. Moreover, the calculation of myogenic index does not rely on a reference diameter.

The intrinsic properties of myogenic response, however, make the identification and analysis of myogenic index more difficult than incremental distensibility. The distensibility curves for passive responses usually show similar patterns; distensibility remains positive but steadily decreases close to zero with the increase of luminal 
pressure. This can be explained by the increase in the slope of the pressure-diameter curves. On the other hand, pressure-diameter curves for myogenic response are more complicated as myogenic indices for arteriolar constriction and dilation are represented by negative and positive values, respectively. Consequently, curves of myogenic index are less characterized than incremental distensibility.

The myogenic index, derived from the pressure-diameter relationship, helps examine the level of arteriolar constriction. However, it is not a definitive mechanical parameter for understanding the mechanics of the myogenic response. Wall tension, physically as the circumferential force per wall thickness, has also been used to characterize the myogenic response $(11,13,20,25,41,47,58,68,78,79)$. It has been proposed as a mechanism for myogenic response from studies of renal autoregulation $(1,67)$. Combined with studies of intracellular calcium concentration, new evidence supports that wall tension, rather than cell length/stretch, serves as a feedback mechanism that regulates the vascular myogenic response $(41,79)$. Other studies argue that the feedback mechanism may not rely on wall tension, but rather on circumferential wall stress (11, 20, 35). Regardless, the mechanics is important.

Based on a strong correlation between wall tension and intracellular calcium concentration/MLC phosphorylation, it is suggested that wall tension plays a critical role in the regulation of myogenic response $(41,79)$. Yet an elevated level of calcium concentration/MLC phosphorylation in the steady state may only reflect an elevation of pressure. The change of vessel size, and hence radius, contributes little to the change of wall tension, which quantitatively is dominated by pressure. Therefore, a "wall tension- 
regulated" mechanism is arguably the same to pressure-regulated mechanism. It does not help to elucidate the underlying mechanism of myogenic response.

Circumferential wall stress reflects circumferential mechanical loading on a vessel wall. Separate studies using arteriole networks from the hamster cheek pouch and rat cerebral vasculature find that wall tension increases with increasing arterial size, but wall stress remains similar in both large and small arterioles $(11,20)$. These studies may suggest that wall stress has an important role for the regulation of myogenic response.

As the myogenic response is a mechanotransduction process, there is a need for a better understanding of the normal mechano-state of the arteriole. In both in vivo and in vitro conditions, longitudinal stress in the vasculature co-exists with the commonly acknowledged circumferential stress $(28,44)$. In vitro studies of cannulated- and perfused- arterioles typically require an axial stretch to straighten the vessel to a more physiological length for its developing spontaneous tone. Since the properties of circumferential stress have been somehow investigated, the magnitude, characteristics and the potential regulatory role of longitudinal stress becomes quite interesting to address. There are very few reports on the characteristics or the effects on myogenic response of axial stress/stretch in the arteriolar wall except anecdotal studies, which are contradictory. It is reported that roughly an extra $25 \%$ stretch over normal testing length for isolated rat cremaster arterioles does not affect the myogenic response or the vasoconstriction to norepinephrine (41). In the same study, an acute increase of intracellular $\mathrm{Ca}^{2+}$ concentration was observed when the axial stretch was applied. However, the increase declines to a level close to baseline within 3 minutes. 
Contradictory results have been reported, though not described in detail, in isolated rabbit coronary microvessels that an extra $40 \%$ stretch increase the myogenic tone (26).

Many questions about the characteristics of arteriolar axial stress are unanswered. As circumferential stress has been suggested to be one of the regulatory mechanisms for myogenic response, the effects of axial stress accordingly need to be questioned. To construct a three dimensional stress analysis, the magnitude and stress-strain relationship of axial stress need to be identified and compared to those of circumferential stress. The roles of axial stress in mechanotransduction in myogenic responses and its correlation to intracellular calcium concentration and MLC phosphorylation are unknown.

Mechanical indices like wall tension and wall stress have been used to characterize myogenic response. In addition, they are proposed as regulatory mechanisms underlying myogenic response. It remains unknown, however, whether the wall tension and wall stress are real mechanisms that guide myogenic mechanotransduction or as calculated parameters they coincidentally appear to be related to the intrinsic myogenic behaviors.

Most myogenic studies do not consider the effects of axial stretch, which affects the fundamental behaviors of microvessels. As previously explained for passive responses (that axial stretch may affect the curves of circumferential stress-strain and the associated elastic modulus as well as the pressure-diameter curves and the associated incremental distensibility), different arteriolar axial stretches are capable of leading to different curves of myogenic index, wall tension and circumferential wall stress, which have been used to characterize myogenic response. Although the effects of axial stretch have been studied for conduit arteries $(38,49,80)$, they have not been clarified for 
arteriolar mechanical behaviors and functional responses. Therefore, a study of the properties of axial stress-strain relationship and the effects on arteriolar functions is necessary. 


\section{CHAPTER III}

\section{METHODS}

\section{Preparation}

Male Sprague-Dawley rats $(170-350 \mathrm{~g}, \mathrm{n}=22)$ were anesthetized with intraperitoneal injections of pentobarbital sodium $(60 \mathrm{mg} / \mathrm{kg})$. The right or left cremaster muscle was quickly exteriorized and excised, and the rats were killed by anesthetic overdose. The cremaster was placed in a cooled $\left(4^{\circ} \mathrm{C}\right)$ dissection chamber filled with physiological salt solution (PSS) containing (in $\mathrm{mM}$ ): $145 \mathrm{NaCl}, 4.7 \mathrm{KCl}, 2 \mathrm{CaCl}_{2}, 1.2 \mathrm{MgSO}_{4}, 1.2$ NaH2PO, 3 MOPS, 5 glucose, 2 pyruvate, 0.02 EDTA and 1\% bovine serum albumin. Segments of second-order arterioles were carefully dissected with sharpened instruments and transferred to a custom-designed $3 \mathrm{ml}$ chamber $(20,22)$. The chamber and cannulation pipettes contained PSS with the same ionic concentration as the dissection buffer. The PSS had a $\mathrm{pH}$ of 7.4 at $37.5^{\circ} \mathrm{C}$ whereas the $\mathrm{pH}$ of the dissection buffer was adjusted to be 7.4 at $4^{\circ} \mathrm{C}$.

At room temperature, both ends of the arteriole were cannulated with micropipettes and secured with 11-O ophthalmic suture $(19,52)$. Both pipettes had tips pulled to $\sim 50$ microns in inner diameter. The right pipette (originally $1.97 \mathrm{~mm} \mathrm{OD}, 1.79 \mathrm{~mm}$ ID; Drummond Scientific, Broomall, PA) was secured in a holder on a V-track microperfusion system (52), whereas the left pipette (originally $1.5 \mathrm{~mm} \mathrm{OD}, 1.0 \mathrm{~mm} \mathrm{ID}$; Garner Glass, Claremont, CA) was bent to a right angle using a microforge and then fit tightly into a compliant silicone tube. After the chamber was transferred to the stage of 
an inverted microscope (19) and the pipette system tested for pressure leaks, a thin bronze wire connected to a force transducer (Model \# 405 A, $10 \mathrm{mN}$ full scale, Aurora Scientific, Aurora, Ontario, Canada) was hooked to the left pipette and affixed using a tiny drop of wax (Fig. 1A).

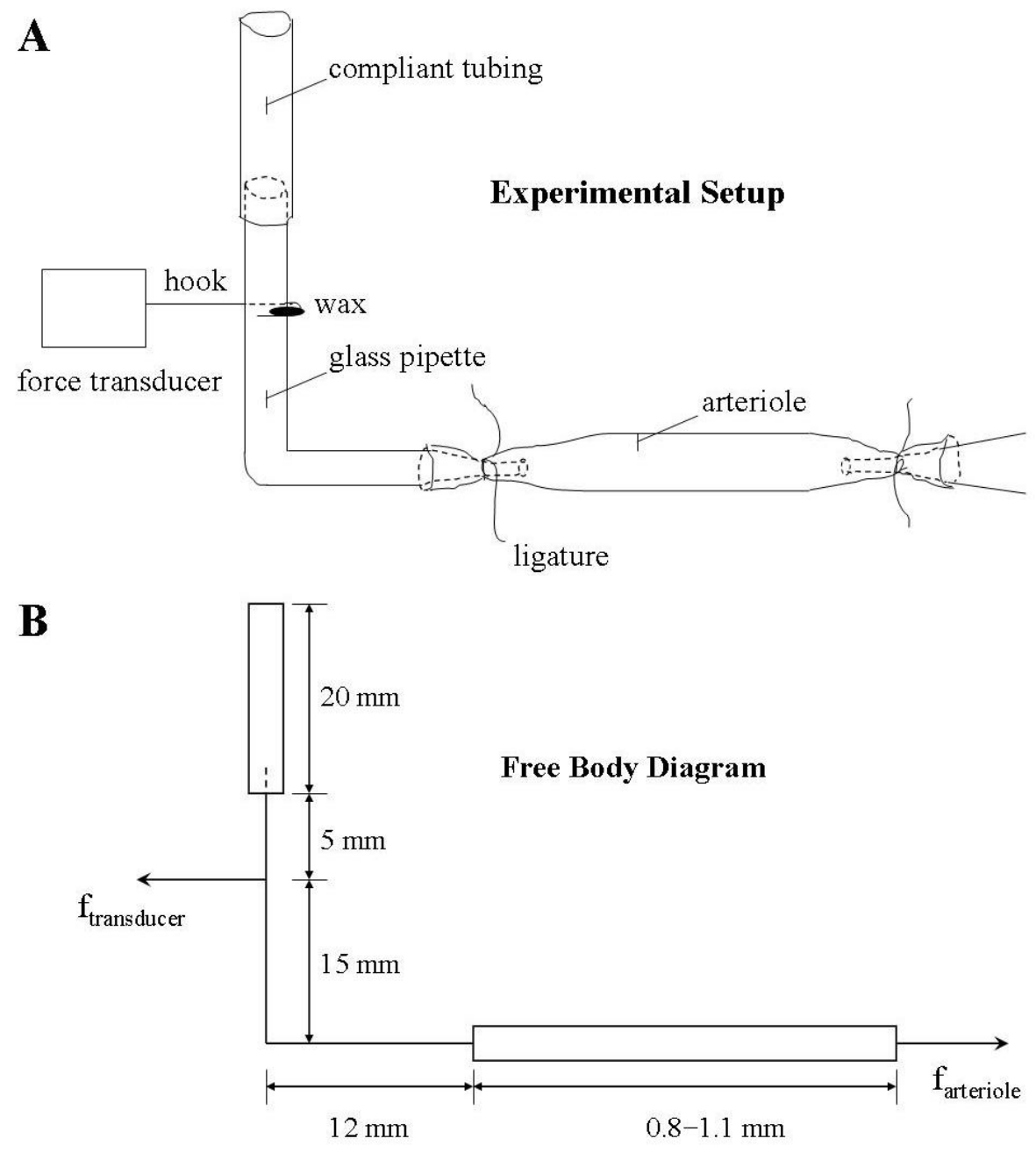

Fig. 1. Experimental setup of the arteriolar biaxial stretch test (A) and corresponding free body diagram (B). A bronze wire connected to the transducer was hooked to the left pipette $5 \mathrm{~mm}$ below its connection to a compliant tube and secured by wax. The actual size of the arteriole was much smaller than shown. $\mathrm{f}_{\text {arteriole }}$ was the arteriolar axial force and the balance of force is illustrated in the free body diagram. 
The compliant tubing allowed the left pipette to move slightly to facilitate a consistent measurement of arteriolar axial force. The tubing-pipette assembly resembled a cantilever, which mainly sustained two horizontal forces, i.e., $\mathrm{f}_{\text {transducer }}$ and $\mathrm{f}_{\text {arteriole }}$, in the leftward and rightward directions, respectively. Calibrations demonstrated that this setup provided a linear relation between the force on the arteriole and that measured by the transducer $\left(\mathrm{f}_{\text {transducer }}=1.573 \times \mathrm{f}_{\text {arteriole }}\right.$, quantitatively consistent with a basic structural analysis, not shown), thus allowing simultaneous measurement of arteriolar axial force and intraluminal pressure in functional vessels (30).

During an experiment, the arteriole was pressurized using a static reservoir and continuously superfused $(0.4 \mathrm{ml} / \mathrm{min})$ with PSS using a roller pump. The chamber, pipette, and force transducer were mounted onto the stage of an inverted microscope and the microscopic image was displayed using a Dage MTI newvicon videocamera. Luminal pressure was monitored by a pressure transducer and arteriolar diameter was measured with a videomicrometer (19).

Experimental protocols

After pressurization to $80 \mathrm{cmH}_{2} \mathrm{O}$, the distance between the two pipette tips was increased by moving the right pipette until the vessel was barely straightened. The internal diameter and arteriolar length were recorded using Zeiss 4x and 16x Achromat objectives, respectively, on the inverted microscope. The initial axial length of the arteriole was measured as the distance between the 2 sutures that secured the vessel to the pipettes and referred to as the reference length $\mathrm{L}_{\mathrm{P}=80}$. Arterioles were then 
equilibrated in a heated bath $\left(35^{\circ} \mathrm{C}\right)$ for at least 30 minutes until they developed spontaneous tone.

Vessels that developed spontaneous tone (a minimal 25\% constriction in lumen diameter) were selected for testing either myogenic responses or norepinephrine (NE) responses. Vessels were examined for myogenic responses by decreasing luminal pressure to $30 \mathrm{cmH}_{2} \mathrm{O}(22.1 \mathrm{mmHg})$, then elevating pressure sequentially to $55,80,100$ and $120 \mathrm{cmH}_{2} \mathrm{O}$ (that is, 40.4, 58.8, 73.5 and $88.2 \mathrm{mmHg}$; note that intraluminal pressure is plotted in all figures in mmHg instead of $\mathrm{cmH}_{2} \mathrm{O}$ ). At each prescribed pressure step and length, the inner diameter and the axial force were measured after 3 minutes of equilibration. A viable arteriole was tested at 3 to 4 different axial stretches between $85 \%$ and $110 \%$ of $\mathrm{L}_{\mathrm{P}=80}$. At a pressure of $80 \mathrm{cmH}_{2} \mathrm{O}$, the length of the vessels was carefully adjusted followed by 5 to 10 minutes of equilibration, after which the vessel was tested again for myogenic responsiveness under the new axial stretch. Each arteriole was usually tested first at its original length, then at a lower axial stretch, and finally at a higher axial stretch such as $110 \%$ of $\mathrm{L}_{\mathrm{P}=80}$.

Vessels selected for testing NE responsiveness were maintained at an intraluminal pressure of $80 \mathrm{cmH}_{2} \mathrm{O}$. With superfusion paused, the internal diameter and arteriolar axial force were recorded 3 minutes after administration of $\mathrm{NE}$ to the vessel bath. Doses were tested cumulatively, with the maximal dose of NE being $10^{-6} \mathrm{M}$. Similar to tests of myogenic responses, NE responses were tested under 3 to 4 different arteriolar axial stretches if the vessel remained viable. Between the tests at different axial stretches, the bath solution was completely exchanged twice with fresh PSS. 
After testing for either myogenic or NE responses, arterioles were allowed to equilibrate for at least 30 minutes in $\mathrm{Ca}^{2+}$-free PSS. Inner diameters and axial forces were then measured for pressures of $30,55,80,100$ and $120 \mathrm{cmH}_{2} \mathrm{O}$. The vessels were equilibrated for 2 minutes at each prescribed pressure step and length. Measurements were then taken under passive conditions for the same axial stretches that were used to assess myogenic or NE responses. For each arteriole, wall volume was calculated at the different axial stretches by recording both the inner and outer diameters at a pressure of $80 \mathrm{cmH}_{2} \mathrm{O}$ in $\mathrm{Ca}^{2+}$-free PSS solution and an average was taken from the volumes as the final wall volume.

A final step was to record the unloaded state of the arteriole. As the arteriolar lumen typically collapsed at zero intraluminal pressure, making measurement of inner diameter difficult and not meaningful, intraluminal pressure was adjusted to 2 to $3 \mathrm{cmH}_{2} \mathrm{O}$ for the unloaded state so that the arteriole was slightly distended to facilitate the recording of lumen diameter. After this, arteriolar axial length was slowly reduced to an axially unloaded state so that the shape of the arteriole became barely straight and the axial force ceased to decline perceptibly. Finally, the unique unloaded internal diameter $\left(D_{0}\right)$, unloaded axial length $\left(\mathrm{L}_{0}\right)$, and unloaded axial force $\left(\mathrm{f}_{0}\right)$, which represents the offset in the transducer, were recorded for each vessel.

\section{Useful metrics}

The circumferential stretch ratio $\left(\lambda_{\theta}\right)$ was used to delineate structural responses of arterioles and was calculated as $\mathrm{D} / \mathrm{D}_{0}$, where $\mathrm{D}$ is the observed arteriolar internal diameter for a given intraluminal pressure. Circumferential (or hoop) stress was 
calculated as $\sigma_{\theta}=\mathrm{PD} / 2 \mathrm{~h}$, where $\mathrm{P}$ is the intraluminal pressure and $\mathrm{h}$ is the deformed wall thickness; $\mathrm{h}$ was measured directly at $\mathrm{P}=80 \mathrm{cmH}_{2} \mathrm{O}$ in $\mathrm{Ca}^{2+}$-free PSS solution and computed based on incompressibility of the wall at all other pressures. For comparison to data in the literature, an incremental stiffness (or, modulus) was calculated as $\Delta \sigma_{\theta} / \Delta \lambda_{\theta}$, where $\Delta \sigma_{\theta}$ is the change of circumferential stress resulting from a change in circumferential stretch $\Delta \lambda_{\theta}$.

Axial stress was computed as $\sigma_{\mathrm{z}}=\mathrm{f} / \mathrm{A}$, where $\mathrm{f}$ is the axial force in the wall of the arteriole and the cross section area of the wall A equals $\pi h(D+h)$. The force $f$ was calculated as $\left(f_{\text {arteriole }}+f_{\mathrm{P}}\right)$, where $f_{\text {arteriole }}$ is the total axial force on the left pipette (Fig. 1B), which was calibrated to be $\left(f_{\text {transducer }}-f_{0}\right) / 1.573 ; f_{P}$ is an "extra" force arising from intraluminal pressurization and equals $\mathrm{P} \pi\left(\mathrm{D}^{2}-\mathrm{D}_{\text {pipette }}{ }^{2}\right) / 4$, where $\mathrm{D}_{\text {pipette }}=50 \mu \mathrm{m}$ is the internal diameter of the pipettes. Axial stretch ratio $\lambda_{z}$ was calculated as $L / L_{0}$, where $L$ is the observed axial length. The stress ratio $\sigma_{\theta} / \sigma_{z}$ was used to characterize the biaxial state of stress experienced by the arteriole.

Incremental distensibility (5) and myogenic index $(19,74)$ were calculated as $100(\Delta \mathrm{D} / \mathrm{D}) / \Delta \mathrm{P}$ for passive responses and myogenic responses, respectively, where $\Delta \mathrm{D} / \mathrm{D}$ is the fractional change in lumen diameter in response to each change in intraluminal pressure $(\Delta \mathrm{P})$. The constriction ratio, defined by normalizing arteriolar diameter by the maximal diameter, was calculated as $\mathrm{D} / \mathrm{D}_{\mathrm{P}=30}$ for myogenic responses and $\mathrm{D} / \mathrm{D}_{\mathrm{NE}=-9}$ for NE responses, where $\mathrm{D}_{\mathrm{P}=30}$ is the arteriolar inner diameter at a pressure of $30 \mathrm{cmH}_{2} \mathrm{O}$ and $\mathrm{D}_{\mathrm{NE}=-9}$ is the inner diameter in response to NE of $10^{-9} \mathrm{M}$. 


\section{Data analysis}

The results were tested for statistical significance using one-way ANOVA followed by post-hoc analysis (Tukey HSD) for multiple-comparisons. Differences were considered significant at the $\mathrm{p}<0.05$ level. 


\section{CHAPTER IV}

\section{RESULTS}

Table 2. Average axial stretch ratios for low, moderate, and high stretch groups

\begin{tabular}{|llll|}
\hline & Low stretch & Moderate stretch & High stretch \\
\hline Passive & $1.31 \pm 0.01(10,8)$ & $1.45 \pm 0.01(11,11)$ & $1.61 \pm 0.02(12,11)$ \\
Myogenic & $1.30 \pm 0.02(8,6)$ & $1.45 \pm 0.02(6,6)$ & $1.61 \pm 0.03(7,5)$ \\
NE & $1.31 \pm 0.01(7,6)$ & $1.45 \pm 0.01(14,12)$ & $1.60 \pm 0.02(12,8)$ \\
\hline
\end{tabular}

Values are means \pm SE. The number of arteriolar axial stretches and the number of tested arterioles are shown, respectively, in parenthesis. Only one vessel was tested for each animal.

Table 3. Morphological characteristics of arterioles in unloaded and pressurized conditions, each in $\mathrm{Ca}^{2+}$-free PSS

\begin{tabular}{|llll|}
\hline & Passive group $(\mathrm{n}=13)$ & Myogenic group $(\mathrm{n}=6)$ & NE group $(\mathrm{n}=12)$ \\
\hline $\mathrm{L}_{0}(\mu \mathrm{m})$ & $661.4 \pm 43.3$ & $647.0 \pm 52.3$ & $611.5 \pm 55.8$ \\
$\mathrm{D}_{0}(\mu \mathrm{m})$ & $66.0 \pm 4.2$ & $56.8 \pm 3.2$ & $59.6 \pm 3.6$ \\
$\mathrm{D}_{\mathrm{P}=80}(\mu \mathrm{m})$ & $130.3 \pm 5.7$ & $126.5 \pm 1.5$ & $126.8 \pm 4.4$ \\
$\mathrm{~h}_{\mathrm{P}=80}(\mu \mathrm{m})$ & $9.8 \pm 0.7$ & $11.0 \pm 1.1$ & $9.9 \pm 0.8$ \\
$\lambda_{0}$ & $1.47 \pm 0.02$ & $1.46 \pm 0.02$ & $1.46 \pm 0.03$ \\
\hline
\end{tabular}

$\mathrm{L}_{0}$ and $\mathrm{D}_{0}$ are arteriolar unloaded length and unloaded diameter respectively. $\mathrm{D}_{\mathrm{P}=80}$ and $\mathrm{h}_{\mathrm{P}=80}$ are arteriolar inner diameter and wall thickness at the initial axial stretch and intraluminal pressure of 80 $\mathrm{cmH}_{2} \mathrm{O} . \lambda_{0}$ is the original axial stretch ratio at which the vessel was tested. Values are means $\pm \mathrm{SE}$. The number of tested arterioles for each response is shown in parenthesis. Only one vessel was tested for each animal.

\section{Morphological data}

For passive and NE responses, the axial stretch ratios were divided into 3 distinct groups, i.e., low $\left(1.25<\lambda_{z}<1.35\right)$, moderate $\left(1.40<\lambda_{z}<1.50\right)$ and high $\left(1.55<\lambda_{z}<1.70\right)$ stretches. Note that very few arteriolar axial stretch ratios fell below 1.25 or arose above 
1.70; stretch ratios that did not fit into the groups were excluded. For myogenic responses, low, moderate, and high stretch were defined as $1.23<\lambda_{z}<1.38,1.40<\lambda_{z}<1.50$ and $1.53<\lambda_{z}<1.70$, respectively, because of a smaller number of samples. The averages for the axial stretch ratios were similar for both groups (Table 2).

Arterioles were similar in size, independent of grouping, in both unloaded and loaded (e.g., axially stretched and pressurized at $80 \mathrm{cmH}_{2} \mathrm{O}$ ) configurations (Table 3). Unloaded lengths and diameters were not significantly different and arteriolar diameters and wall thicknesses were comparable at a pressure of $80 \mathrm{cmH}_{2} \mathrm{O}$. Based on statistical tests, arterioles were stretched roughly to the same degree $\left(\lambda_{0}\right)$ when the protocols were started.

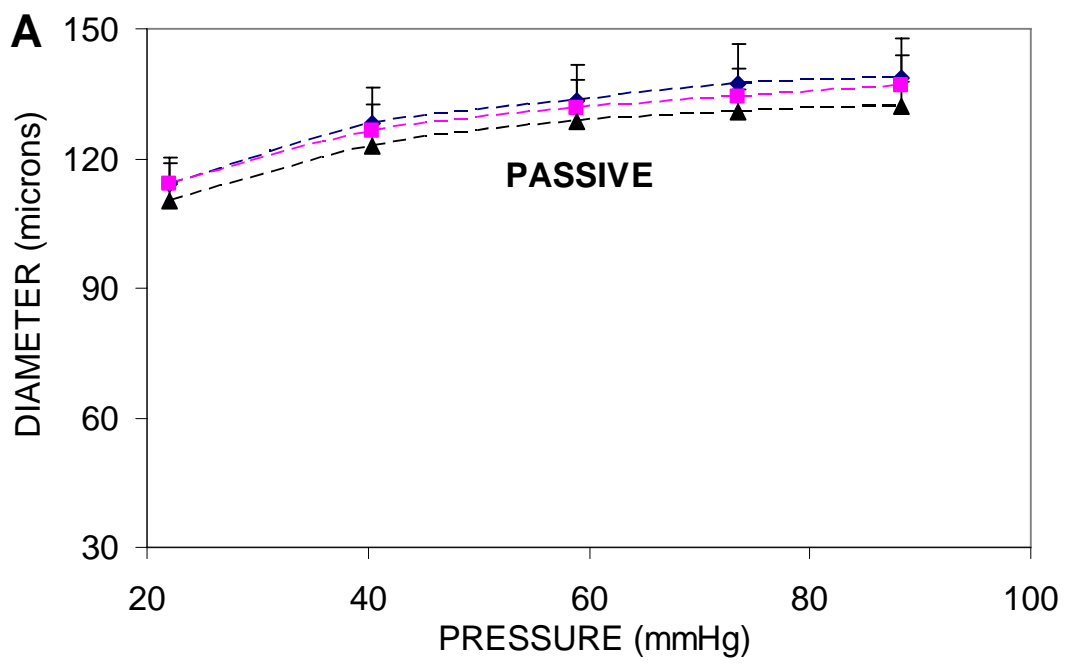

Fig. 2. Change in diameter as a function of intraluminal pressure (A, B) or NE concentration (C) at low (diamonds), moderate (squares), or high (triangles) axial stretch. Intraluminal pressure was kept at 80 $\mathrm{cmH}_{2} \mathrm{O}$ for tests of $\mathrm{NE}$ responsiveness. Values are means $+\mathrm{SE}$. Values at high stretches that were significantly different from both low and moderate stretches are indicated by $(*)$. Values at high stretches that were significantly different from either moderate stretches $(* *)$ or low stretch $(* * *)$ are also indicated. 

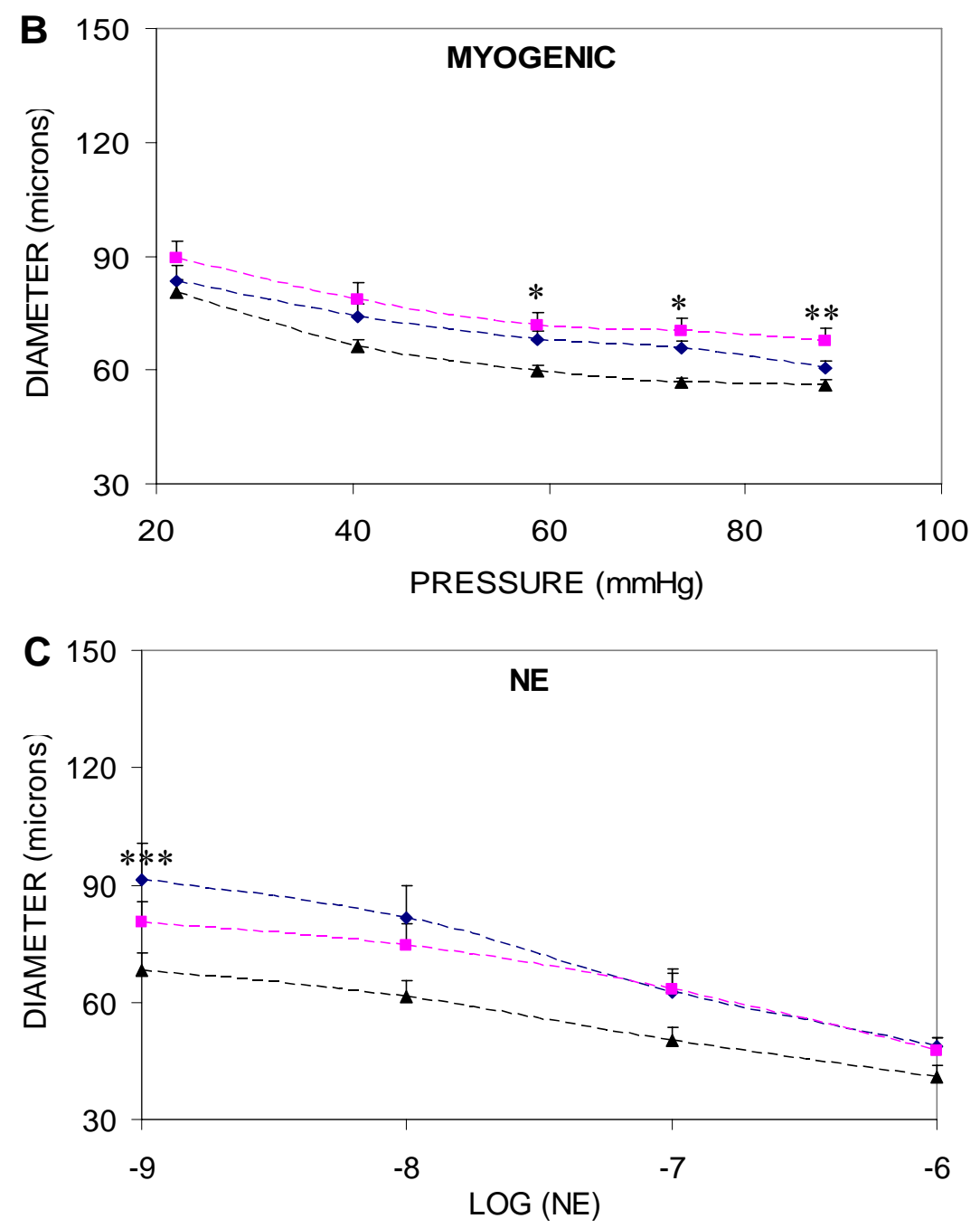

Fig. 2. Continued.

Diameter vs. pressure and NE

Relationships between diameter and intraluminal pressures as well as concentration of NE reflect the structural stiffness of the vessel; they are shown in Figure 2. Vessels at low, moderate, and high stretch ratios all developed myogenic responses (Fig. 2B) and constricted to NE (Fig. 2C). Not surprisingly, one effect of the high stretch ratio was a smaller diameter at each pressure in the passive state. Indeed, the same was true for 
myogenic and NE responses, hence the diameters were smaller due to the effect of axial elongation. Note the statistically significant effect of high axial stretch on the myogenic response at pressure $\geq 60 \mathrm{mmHg}$.
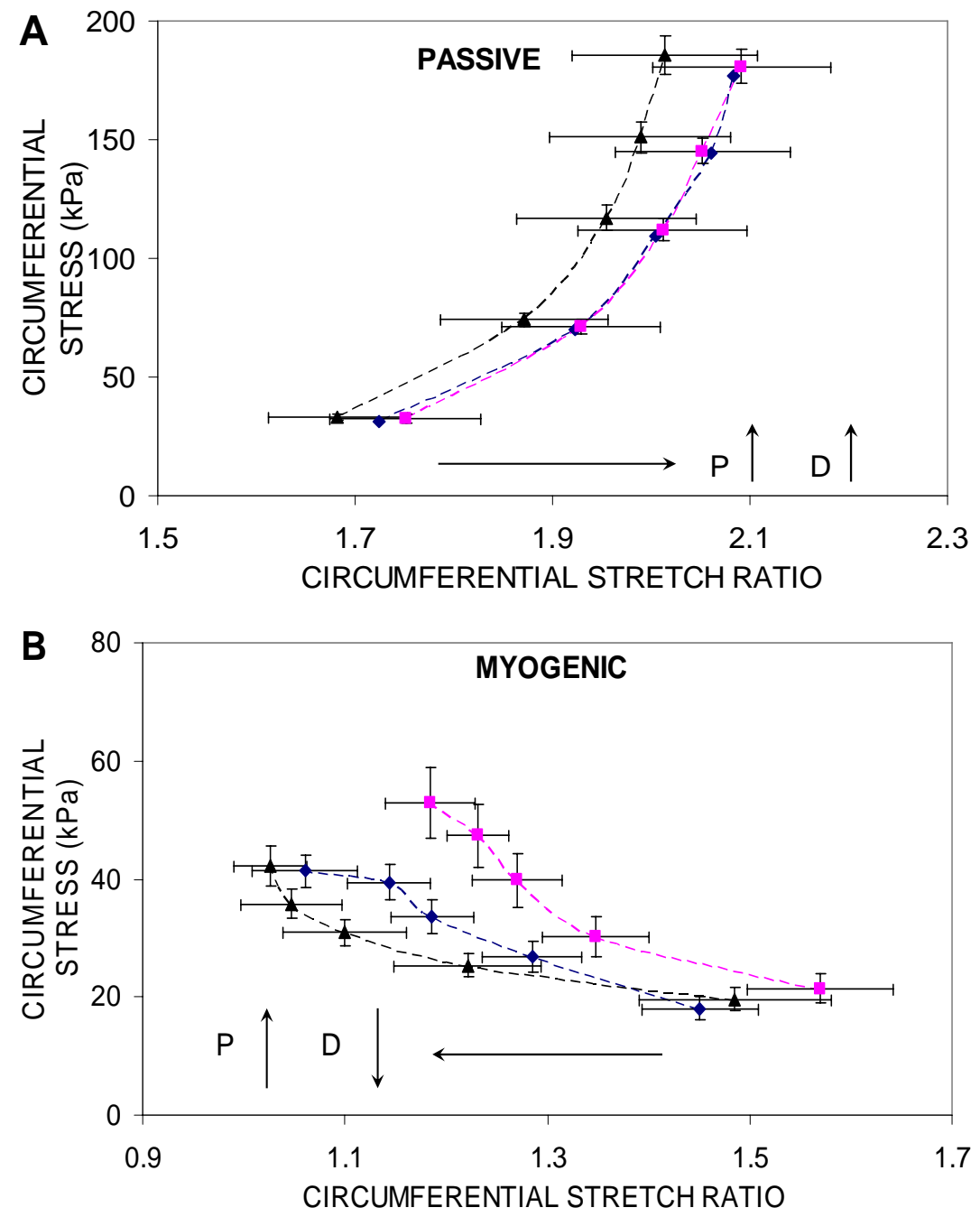

Fig. 3. Relationship between circumferential stress $\sigma_{\theta}$ and circumferential stretch ratio $\lambda_{\theta}$ for passive (A), myogenic (B), and NE (C) responses at low (diamonds), moderate (squares), or high (triangles) axial stretch. Increases or decreases in the intraluminal pressures, inner diameters, and dose of NE are illustrated with upward or downward arrows, respectively, for changes in the $\lambda_{\theta}$ indicated by the direction of the horizontal arrow. Values are means \pm SE. 




Fig. 3. Continued.

Circumferential stress vs. circumferential stretch ratio

Relationships between circumferential stress and circumferential stretch reflect the material properties of the wall (Fig. 3). For passive responses, high axial stretch decreased the arteriolar luminal diameter and accordingly lowered the circumferential stretch ratio, which led to a leftward shift of the circumferential stress-stretch curve while preserving the shape of the curve (Fig. 3A); this change was not statistically significant, however. Not surprisingly, the associated incremental stiffnesses at different axial stretch ratios were also not significantly different from each other (e.g., $~ 5.4 \mathrm{MPa}$ at a moderate stretch at an intraluminal pressure of $80 \mathrm{cmH}_{2} \mathrm{O}$ ).

Stress-stretch curves for passive and myogenic responses were very different in character. The passive response was highly nonlinear, similar to that of arteries, whereas relationships for the myogenic and NE groups were nearly linear. Because the increase 
in pressure during myogenic responses numerically played a greater role than the consequential decrease in lumen diameter in the calculation of circumferential stress, this stress increased with intraluminal pressure even though lumen diameter decreased (Fig. 3B). Compared to passive responses, arteriolar lumen diameters during myogenic responses were much smaller while wall thicknesses were much greater at the equivalent intraluminal pressures. Therefore, circumferential stresses were smaller based on the law of Laplace.

As illustrated in Figure 2, the ranges of circumferential stretch ratios were different between passive and active responses. As lumen diameters were smaller during active responses, i.e., myogenic and NE responses, circumferential stretch ratios were accordingly smaller. High axial stretch slightly flattened the myogenic stress-strain curve (Fig. 3B), unlike passive responses in which the shape of the stress-stretch curve was almost unaffected (Fig. 3A). Again, there was a statistically significant effect of high axial stretch at pressures $\geq 60 \mathrm{mmHg}$.

Stress-stretch curves for NE responses were measured while intraluminal pressure was kept constant at $80 \mathrm{cmH}_{2} \mathrm{O}(58.8 \mathrm{mmHg})$. It is interesting that the stress-stretch relationships at low, moderate, and high stretch levels all appeared fairly linear and very similar (Fig. 3C). The stress ranges for the NE groups were similar to those during myogenic responses. Compared to passive responses, arterioles during both myogenic and NE responses maintained their stress at a much lower level, i.e., around 20 - $60 \mathrm{kPa}$. 

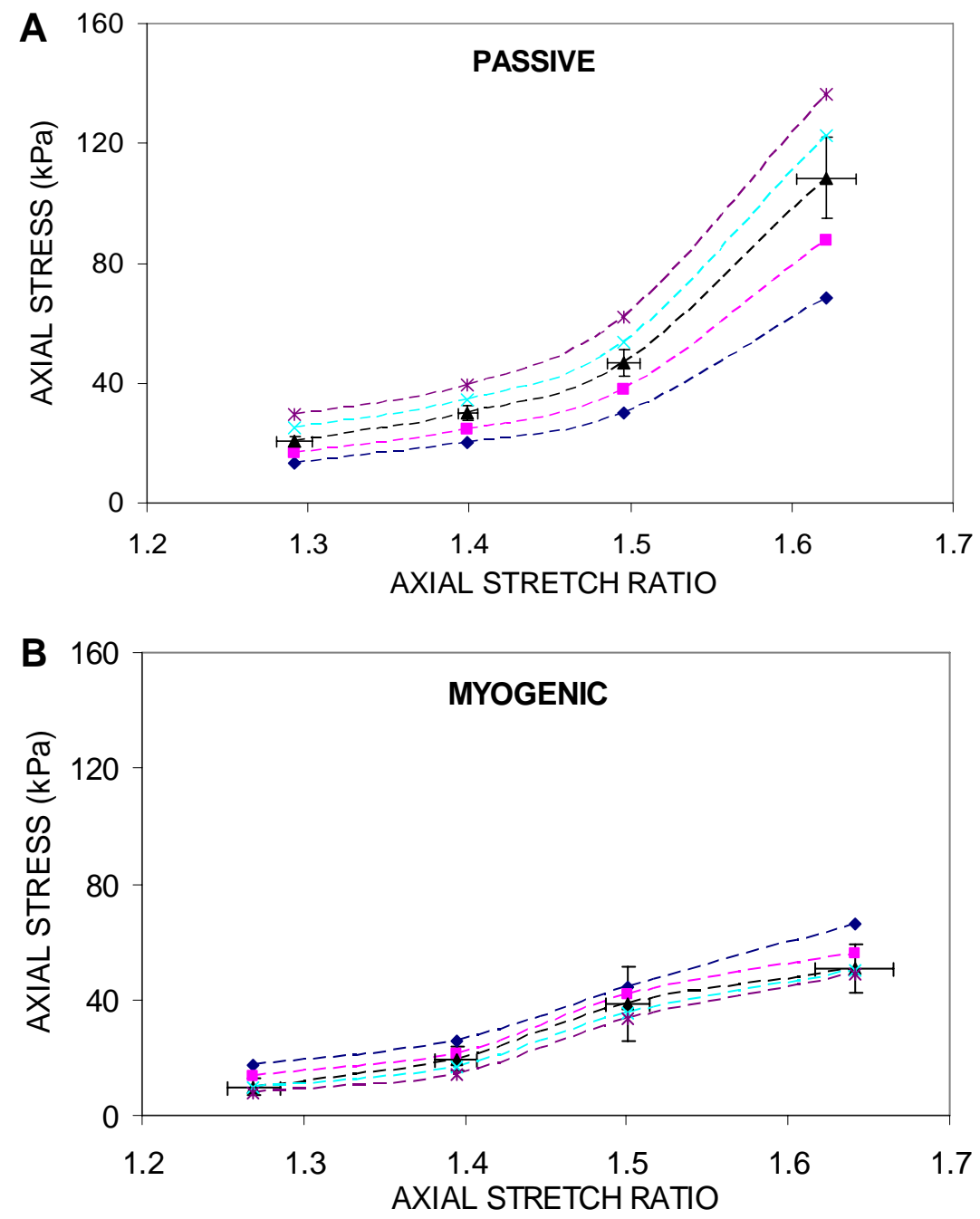

Fig. 4. Relationship between axial stress $\sigma_{z}$ and axial stretch ratio $\lambda_{z}$ for passive (A), myogenic (B) and NE (C) responses. Panels A and B show data at constant pressures of 30 (diamonds), 55 (squares), 80 (triangles), 100 (crosses), and 120 (asterisks) $\mathrm{cmH}_{2} \mathrm{O}$. Panel $\mathrm{C}$ shows data at $\mathrm{NE}$ concentration of $10^{-9}$ (diamonds), $10^{-8}$ (squares), $10^{-7}$ (triangles), and $10^{-6}$ (crosses) M. For clarity, means $\pm \mathrm{SE}$ are only shown for intraluminal pressure of $80 \mathrm{cmH}_{2} \mathrm{O}$ for passive and myogenic responses, and for $\mathrm{NE}$ of $10^{-6}$ M. 


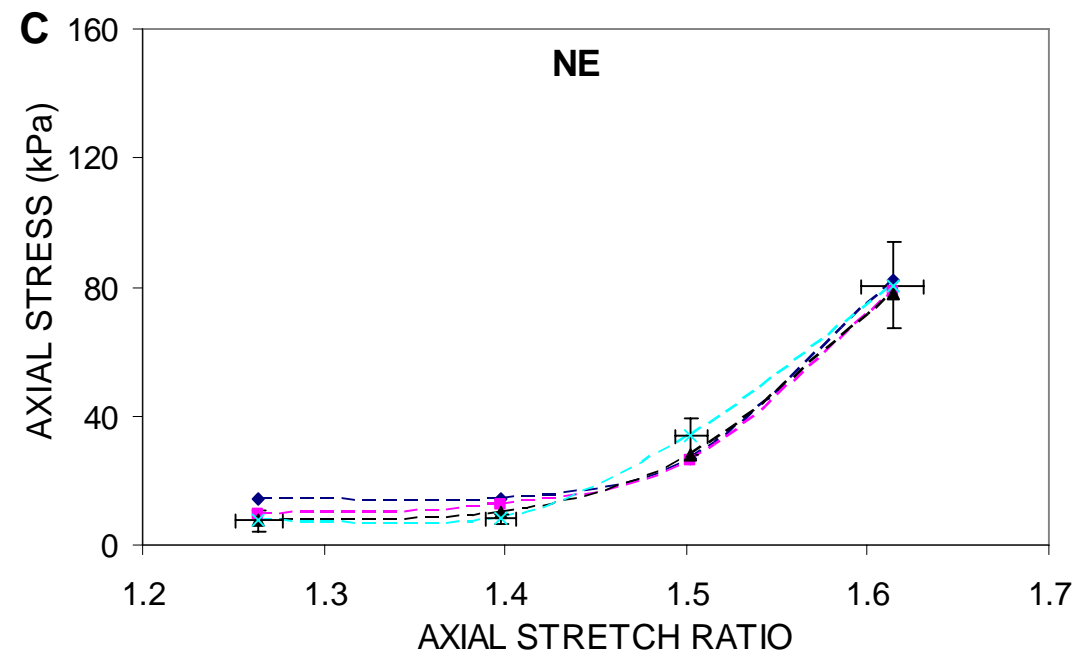

Fig. 4. Continued.

Axial stress vs. axial stretch ratio

As data for three different groups of axial stretch ratios (Table 2) might not adequately illustrate the characteristics of the axial stress-stretch curves, test results at all axial stretch ratios were included and categorized into 4 groups (Fig. 4). Increases in luminal pressure led to an upward shift of the stress-stretch curve for passive responses (Fig. 4A), which demonstrated the effect of the "extra force" $f_{P}$ (recall Fig. 1). Due to the diminished axial "extra-force" during vasoconstriction, the axial stress curve shifted downward for myogenic responses while intraluminal pressure increased (Fig. 4B). When intraluminal pressure was low, the axial stress during the myogenic response was comparable to that of the passive response. When intraluminal pressure was elevated, however, axial stress was much lower if arterioles were regulated by myogenic constriction. 
Finally, the axial stress-stretch curves for the NE responses were similar to those for the passive responses at a pressure of $80 \mathrm{cmH}_{2} \mathrm{O}$, the same intraluminal pressure that was maintained for all tests of NE responses. The stress-stretch curves were not changed except at high doses of NE, e.g., $10^{-6} \mathrm{M}$ (Fig. 4C).
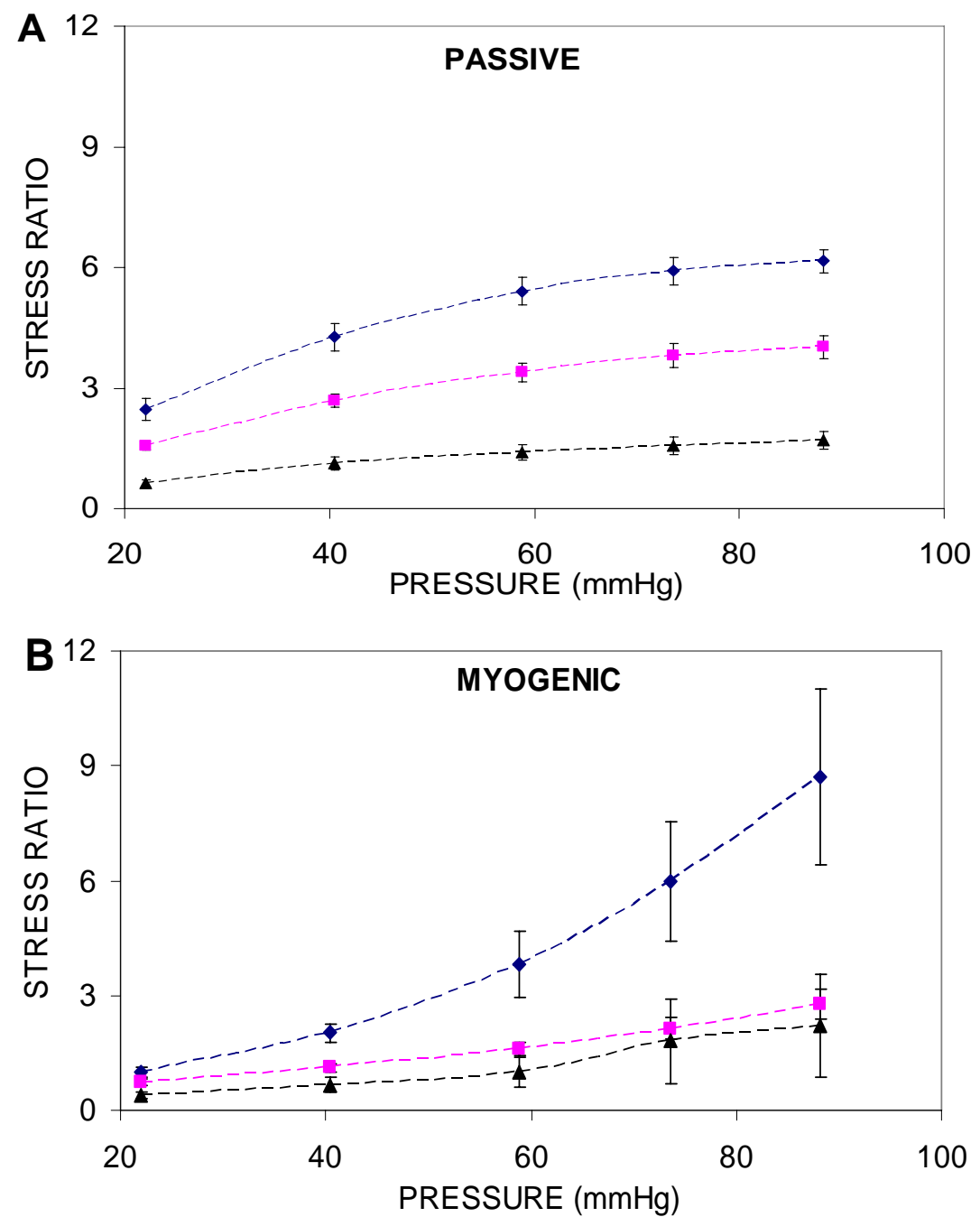

Fig. 5. Change of stress ratio $\sigma_{\theta} / \sigma_{z}$ as a function of intraluminal pressure (A, B) or NE (C) at low (diamonds), moderate (squares), and high (triangles) levels of axial stretch. Values are means $\pm \mathrm{SE}$. 


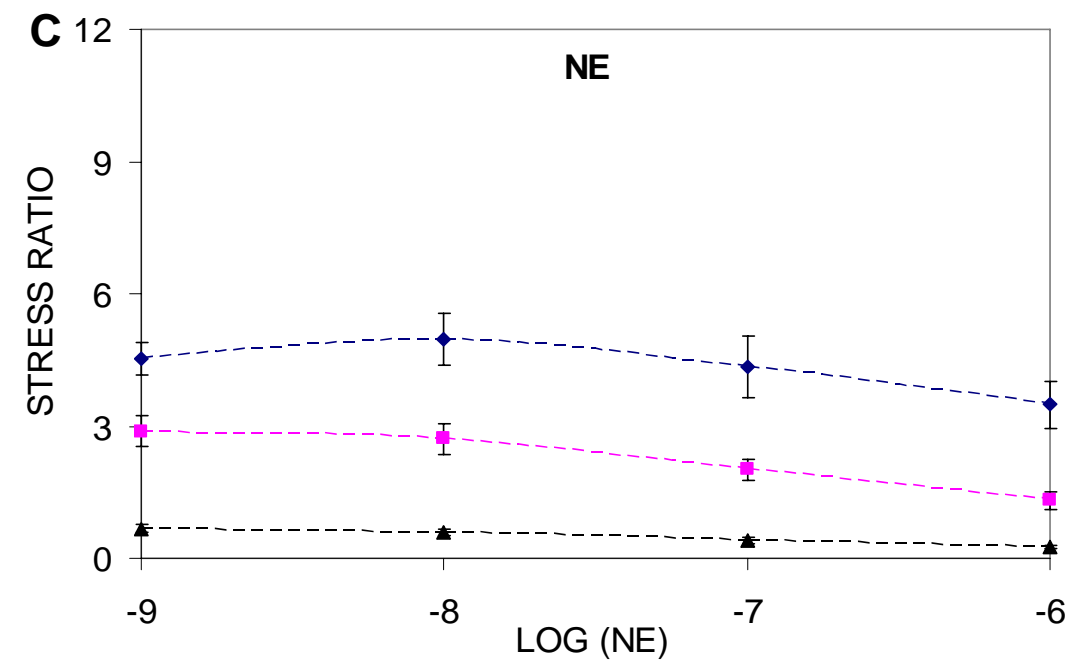

Fig. 5. Continued.

\section{Stress ratio vs. pressure and $N E$}

The stress ratio $\sigma_{\theta} / \sigma_{\mathrm{z}}$ represents the inplane biaxial state of stress in the arteriolar wall (Fig. 5). Since the stress ratio was calculated as the ratio of circumferential stress to axial stress, the stress ratio curves shifted downward when the axial stretch ratio was increased. Stress ratios for both passive and myogenic responses increased slowly with elevation of intraluminal pressure at low and moderate axial stretches (Fig. 5A and Fig. 5B), but changed little with pressurization at high axial stretch. On the other hand, stress ratios decreased slightly at low and moderate axial stretches in response to higher doses of NE (Fig. 5C), but again changed little at high axial stretch. Although the arteriolar wall sustained large changes in its stress state after axial elongation, it appeared to maintain functional responses. 

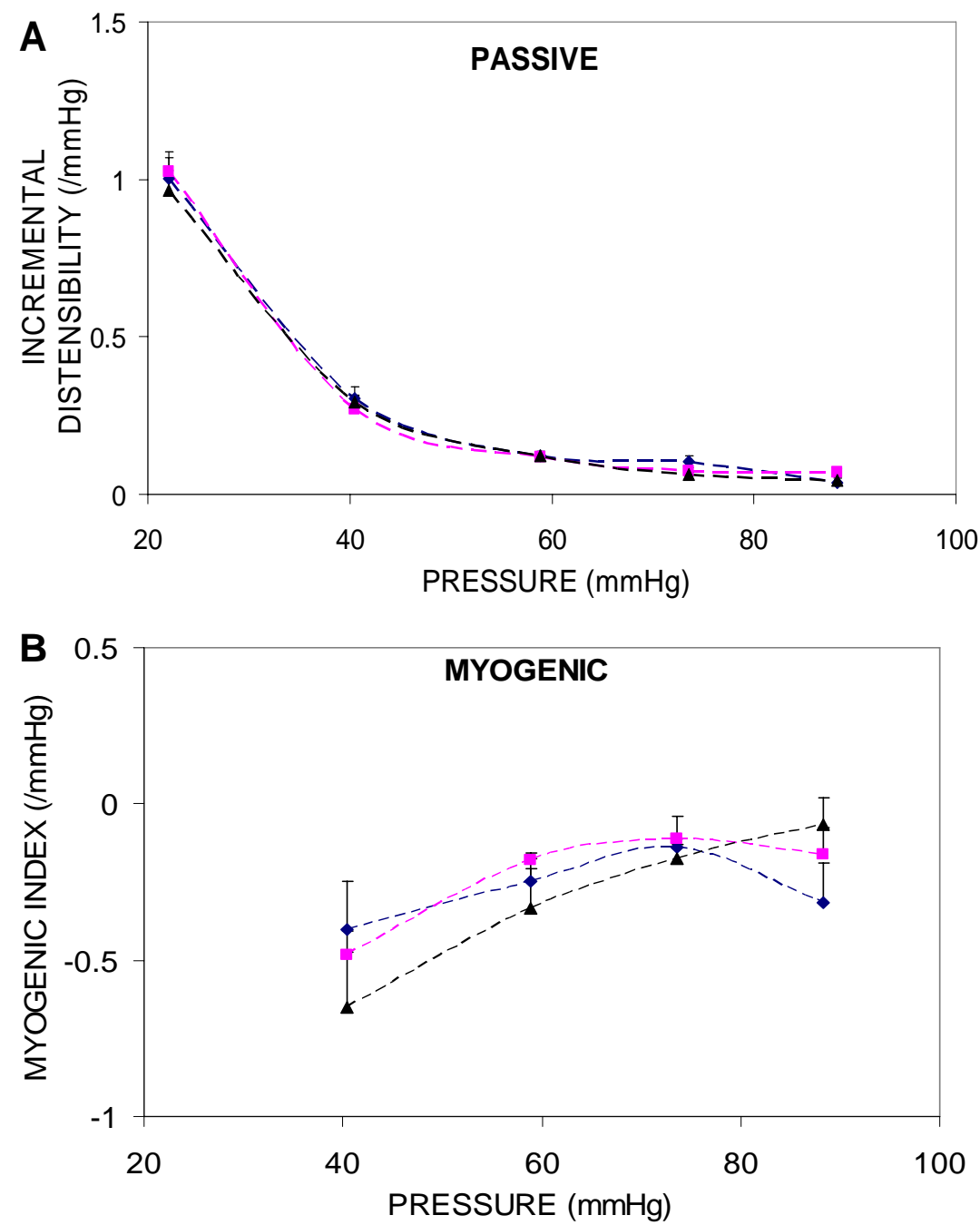

Fig. 6. Incremental distensibility (A) and myogenic index (B) at low (diamonds), moderate (squares) and high (triangles) axial stretch. Values are means + SE. No significant difference was found $(p<0.05)$.

Incremental distensibility, myogenic index, and constriction ratio

Incremental distensibility, determined by the slope of the pressure-diameter relationship via $100(\Delta \mathrm{D} / \mathrm{D}) / \Delta \mathrm{P}$, describes the structural behavior of arterioles in the passive condition. As the pressure-diameter curves at multiple axial stretches largely paralleled or overlapped each other (Fig. 2A), the curves of incremental distensibility 
likewise overlapped (Fig. 6A). Note, too, the near constancy of distensibility at $\mathrm{P} \geq 60$ $\mathrm{mmHg}$.

The myogenic index is given by the same formula and has the physical meaning, i.e., changes of diameter in response to changes in intraluminal pressure. As the arterioles showed consistent constriction in response to increased luminal pressure within our test range, the myogenic index was always negative. Note, too, that from around $40 \mathrm{mmHg}$ to around $75 \mathrm{mmHg}$, the gradual decrease in myogenic constriction reflected characteristics of the curvatures in Figure 2B.

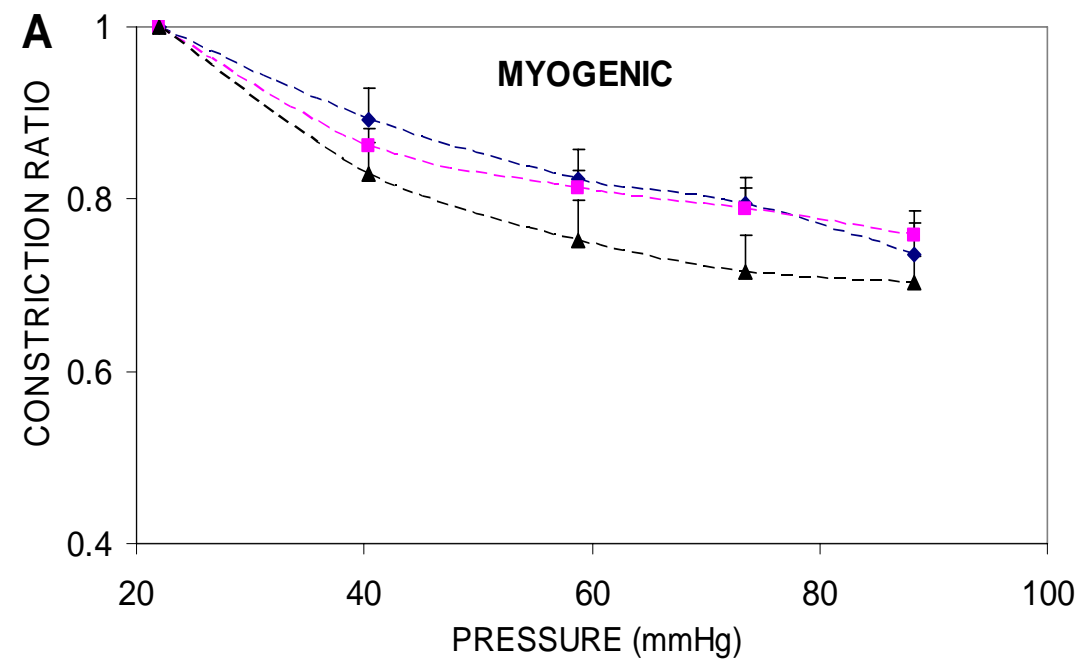

Fig. 7. Change of constriction ratio as a function of intraluminal pressure (A) or NE (B) at low (diamonds), moderate (squares) and high (triangles) axial stretch. Values are means + SE. No significant difference was found $(\mathrm{p}<0.05)$. 


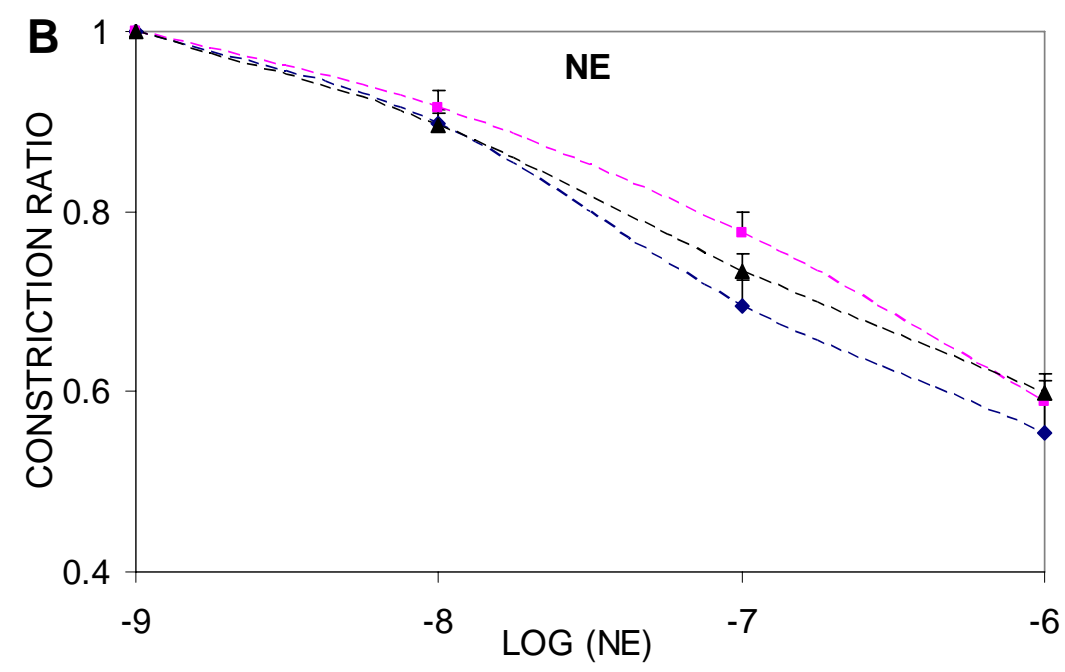

Fig. 7. Continued

The constriction ratio was used to characterize and compare the level of active response, i.e., myogenic (Fig. 7A) and NE (Fig. 7B) responses, by normalizing the current diameter to the baseline diameter (i.e., $\mathrm{D}_{\mathrm{P}=30}$ for myogenic response and $\mathrm{D}_{\mathrm{NE}=-9}$ for NE response). As seen from Fig. 7, the constriction ratios decreased monotonically as a function of increased intraluminal pressure and concentration of NE. Although differences due to altered axial stretch were not significant, there was a trend toward the greater myogenic response at the high stretch. Statistically, however, an optimal axial stretch for smooth muscle contractility was not obvious over the range studied. Finally, the constriction induced by NE was nearly linear in terms of concentration and it reached greater levels than that induced by myogenic response. 


\section{CHAPTER V}

\section{DISCUSSION}

Quantifying the biomechanics of arterioles is essential for understanding both their role in local control of blood pressure and flow (i.e., resistance) and their adaptation to altered mechanical stimuli. A related example is the seminal study of Leung et al. (57) on the effects of mechanical stretch on the rate of production of collagen by vascular smooth muscle cells. More recent examples are the effects of mechanical stretch on growth factor production (77), altered cytoskeletal structure (14), and recruitment of focal adhesions $(18,33)$. It is now clear that we must know the normal biomechanical state experienced by a blood vessel and how altered hemodynamic loads perturb the vessel from this normal state.

Similar to the early history of arterial mechanics, prior studies of arteriole physiology and mechanics have focused primarily on circumferential properties, often interpreted in terms of circumferential stress $(7,11)$, wall tension $(1,41)$, distensibility $(5,8)$, growth index (55) etc. Recent findings on arteries suggest, however, that axial loads may be equally as important as circumferential loads in defining the normal biomechanical state (e.g., stress and stretch). It appears, for example, that the normal in-plane state of stress in the arterial wall may be nearly equibiaxial, with values around $\sigma_{\theta} \sim \sigma_{\mathrm{z}} \sim 100 \mathrm{kPa}$ (45). Moreover, when perturbed from their normal axial extension, arteries appear to remodel quickly in order to restore the axial stretch or stress toward baseline $(15,31,38,49)$. A goal of this work, therefore, was to explore the effects of a range of in-plane biaxial 
loads (i.e., axial as well as circumferential) on the passive and active behavior of arterioles under well-controlled conditions in vitro.

Using methods of microvessel cannulation and perfusion originally developed by Duling (25), arterioles are typically stretched axially to a reference length determined by experience and then subjected to functional or mechanical protocols $(3,19,41,55,79)$. That is, there has been no rigorous standard for setting the axial length. A few studies have addressed the role of axial stretch on arteriolar reactivity, but only in an anecdotal manner. For example, it has been reported that a roughly $25 \%$ increase in stretch over the normal testing length did not affect the myogenic response or vasoconstriction response to norepinephrine in isolated rat cremaster arterioles (41).Although an acute increase in intracellular $\mathrm{Ca}^{2+}$ concentration was observed when axial stretch was applied, it declined to a level close to baseline within 3 minutes. Alternatively, it has been reported, though not in detail, that an extra $40 \%$ stretch increased the myogenic tone in isolated rabbit coronary microvessels (26) and that axial stretch affected the contraction force of lymphatic vessels (59). In none of these cases was the axial force recorded, however, and there was no attempt to define axial stretch relative to a unique reference. Our experiments address both of these gaps. Based on the present study, we recommend that a true unloaded axial length $\mathrm{L}_{0}$ should be recorded. In this way, the overall stretch ratio (i.e., extension) can be written as $\lambda_{z}=\lambda_{z}{ }^{*} \Lambda_{z}$, where $\lambda_{z}{ }^{*}$ is the ratio of the current length (L) to the commonly adopted test length (e.g, $\mathrm{L}_{\mathrm{P}=80}$ ) and $\Lambda_{\mathrm{Z}}$ is the ratio of the commonly adopted length to the true unloaded length $\left(\mathrm{L}_{0}\right)$. This would facilitate comparison between laboratories. 
Incorporating a micro-force transducer within a prior experimental system (19) allowed us to measure the axial force experienced by arterioles at different axial extensions while assessing arteriolar reactivity and passive responses to pressure (30). Although the transducer was commercially available and robust (e.g., no hysteresis), there was need for great experimental care. Calibration studies revealed that it was important to apply only a minimal amount of wax to connect the force transducer to the cannula. Another critical issue that minimized measurement error was to maintain a constant depth of the bath solution throughout the experiment as fluctuations in bath level led to changes in loading on the cannula-arteriole lever assembly (Fig. 1). Furthermore, the axial force needed to be measured at a consistent and constant temperature $\left(35^{\circ} \mathrm{C}\right)$. Once these factors were determined in pilot tests, care was exercised to maintain proper conditions. As in biomechanical studies on arteries, it is convenient to use a reference configuration that is unloaded (zero pressure and zero axial force), or nearly so, for calculating strain. Yet, because the arteriolar lumen collapsed at an intraluminal pressure of zero, a low but slightly positive intraluminal pressure that opened the lumen was imposed for recording the reference luminal diameter (48). Unloaded length was determined by slowly moving the pipettes together until the decrease in axial force stabilized; at that point the arteriole appeared to be just straight. Unloaded force was then determined at the unloaded length. Although our objective was to record a unique reference configuration for each isolated arteriole, measurement of the unloaded state always entailed some subjectivity. As the unloaded state was recorded after testing active and passive responses at different stretch levels, the unloaded 
diameter or length may have been over-estimated due to effects of creep or stress relaxation, particularly in the axial direction after high axial stretch. Therefore, the axial stretch may have been slightly under-estimated. Such limitations are common to all such tests due to the history dependence.

Arteriolar axial stretch levels were selected between $85 \%$ to $110 \%$ of the original test length $\left(\mathrm{L}_{\mathrm{P}=80}\right)$. It was found in pilot tests that arterioles stretched less than $85 \%$ of the original value would bend at higher intraluminal pressures such as 100 and $120 \mathrm{cmH}_{2} \mathrm{O}$. Conversely, when axial stretch was over $110 \%$ of the original value, axial force increased rapidly and the arteriole underwent overt deformation. Thus, we decided not to stretch the arterioles further. Similarly, the range of intraluminal pressures and doses of $\mathrm{NE}$ were chosen carefully to avoid higher pressures (e.g., $>120 \mathrm{cmH}_{2} \mathrm{O}$ ) and $\mathrm{NE}$ concentrations (e.g., $>10^{-6} \mathrm{M}$ ) that cause irreversible effects.

Figures $3 \mathrm{~A}$ and $4 \mathrm{~A}$ reveal that the passive biaxial behavior of arterioles is very similar to that of arteries (44). Both the circumferential and the axial stress-stretch responses are highly nonlinear, but differences in character reveal a strong anisotropy (e.g., the axial response is more bilinear with greater compliance at lower extensions). Moreover, increasing axial stretch tends to decrease the circumferential distensibility whereas increasing pressure has less effect on the axial behavior. Finally, at a pressure of $\sim 60$ $\mathrm{mmHg}$ and an axial stretch of $\sim 60 \%$, relative to an unloaded configuration, the passive biaxial state of stress is $\sim 100 \mathrm{kPa}$, similar to that of arteries (45). It should be noted that this strong similarity with arteries was manifested despite a basic difference in testing procedure. Whereas passive arteries are typically tested under cyclic loading (and thus 
first preconditioned) (27), the tests herein were performed via incremental loading. Specifically, passive responses of arterioles were tested following equilibration in $\mathrm{Ca}^{2+}$ free PSS for at least 30 minutes. We did not precondition (as is common in cyclic tests on arteries to reduce hysteresis) before passive testing because the quasi-static incremental loading strategy allows short term stress relaxation prior to data collection. In both classes of tests, therefore, viscoelastic effects are minimized.

Similar to the strongly anisotropic passive behavior, Figures $3 \mathrm{C}$ and $4 \mathrm{C}$ reveal a strongly anisotropic behavior when the vessels were contracted with NE. The linear circumferential response over the range of stretches considered is in stark contrast to the nearly bi-linear axial response. In particular, similar to the passive response, an axial stretch $\sim 50 \%$ appeared to be that point at which the axially-oriented adventitial collagen began to straighten and thus stiffen the vessel markedly. We note that the in vivo state of many soft tissues tends to be near such "elbows" in the stress-stretch response, an issue that we revisit below.

Finally, Figures 6 and 7 suggest that myogenic responsiveness was not altered significantly by the range of axial forces applied in our study. We considered two different metrics of myogenic reactivity: the myogenic index (Fig 6), which has been commonly used to characterize and quantitate myogenic vasoconstriction, and a constriction ratio, which represents the constrictive characteristics of arteriolar lumen and facilitates direct comparison between pressure-induced (myogenic) and agonistinduced (NE) vasoconstrion. In both cases, there were no significant differences between the responses of vessels at the 4 levels of axial stretch between 0.85 and 1.1 of our 
reference value. These results are in apparent contradiction to anecdotal reports in the literature that axial stretch enhances myogenic reactivity (26) or lymphatic amplitude/frequency changes with pressure (59). Other studies have shown, however, that an assumable $25 \%$ (though the estimate of a $25 \%$ increase in length resulting from a $25 \%$ decrease in diameter is not accurate) increase of axial length in arterioles does not make a significant difference in the levels of myogenic constriction and the response to NE (41). It could be argued that we failed to test a sufficient range of axial lengths in our protocols, but, we used a range of axial loads that encompassed those used in our lab for over 20 years. Stretching below 0.85 of $\mathrm{L}_{\mathrm{P}=80}$ leads to buckling of the vessels at high intraluminal pressures and makes inner diameter measurements difficult and inaccurate. Stretching over 1.1 of $\mathrm{L}_{\mathrm{P}=80}$ leads to an overt deformation of the arterioles and the abrupt increase in axial force would approach the limit of the force transducer Obviously it would have been possible to stretch the vessels axially to lengths that would have produced obvious damage, but such procedures would not be used by experienced investigators wanting to preserve normal vascular reactivity. Another possibility for the apparent discrepancy between our results and others $(26,59)$ is that previous authors may have been referring to transient responses (e.g. pressure-induced constrictions) that were not maintained at the 3 min measurement time used in our study. Alternatively, they may have been referring to the speed at which pressure-induced constriction/dilation occurred. Neither of these parameters was measured in our study.

Note, too, that the achieved circumferential and axial stresses both appear to be $\sim 30$ to $50 \mathrm{kPa}$ at pressures of $\sim 60 \mathrm{mmHg}$ and axial stretches $\sim 60 \%$ relative to an unloaded 
reference. Finally, it is noted that unlike arteries, the operative range of circumferential stretch differs dramatically between the passive (Fig. 3A) and active (Fig. 3B and 3C) behaviors. Likewise the values of circumferential stress are much less in the active state. Both of these differences are due, in large part, to the strong constriction by the arterioles. Our in vitro results thus likely provide bounds for the behavior expected in vivo under basal tone.

As noted earlier, mounting evidence suggests that the normal biomechanical state of an artery correlates well with a nearly equibiaxial intramural stress, and perturbations from normal axial extensions induce rapid and dramatic remodeling processes that appear to seek to restore the normal state. Perhaps because of historical precedence, the physiology and biomechanics of arterioles has been studied primarily in terms of circumferential responses with little attention to the axial direction or its possible affect on the circumferential direction. We found that changes in axial stretch from $85 \%$ to $110 \%$ of a commonly used reference for arteriolar tests (often from $\sim 31$ to $60 \%$ stretch relative to an unloaded reference) induced modest changes in the circumferential and axial stress-stretch responses (Figures 3 and 4) and little change in overall distensibility and myogenic index (Figure 6). Nevertheless, as expected, changes in axial stretch caused significant changes in the biaxial state of stress at a given pressure. In particular, Figure 5 revealed that the ratio of circumferential to axial stress tended to vary little as a function of pressure and contractility when the arterioles were maintained at the high stretch. We conclude, therefore, that if arteries do in fact prefer a nearly equibiaxial state of stress (i.e., if the intramural cells modify wall geometry, structure, and properties 
when perturbed from such a state of stress) and if arterioles have similar mechanobiological controls, then accurately measuring and controlling axial stretch in biomechanical tests on arterioles demands careful attention. In particular, our results suggest that axial extensions used in prior studies based on experience (e.g., the lack of bending of the vessel when pressurized to a target value) may well have been below the preferred value. Although our results show that this may not have markedly affected acute results, they suggest that much more care needs to be given to the degree of axial extension used in many in vivo (e.g., cheek pouch models) and ex vivo (e.g., perfused organ culture) studies. Moreover, because mechanobiological cell-matrix and cell-cell interactions are governed largely by conformational changes by membrane surface receptors (integrins and cadherins), which in turn would be affected by the biaxial state of stress, there is a need to include such considerations in future studies. 


\section{CHAPTER VI}

\section{CONCLUSIONS}

The significance of this study is three-fold. Firstly, it was the first study to systemically investigate the effects of biaxial loading on the mechanical behaviors as well as functional responses of arterioles. The magnitude, characteristics, and influences on active axial stress-stretch relationships had not been explored before this study. Secondly, this study demonstrated that arterioles, surprisingly, maintain their acute regulatory capacity under a large variance in axial stretch levels, which was unexpected as it was speculated that there would be an optimal length at which arterioles have a maximal or somehow distinct pattern of response to physiological agonists, e.g.., pressure and vasoconstrictor. As a result, this finding largely indicates the versatility and strong competence of arterioles, which distinguish them from large arteries. Thirdly, this study was conducted from a view point of quantitating arteriolar mechanical and functional response, in this way, biomechanical modeling of arterioles will be facilitated.

In the future, the studies of arteriolar mechanics can be advanced in several ways. Using the same experimental method, the effects of many diseases or degenerative conditions such as age, hypertension, diabetes, etc on the arteriolar mechanical and functional properties can be investigated. More specifically, the effects may be demonstrated by the shifting of stress-stretch curves, and especially, the change of stress ratio, which may indicate remodeling of arterioles in the axial direction. Accordingly, further study can be focused on how vascular components, e.g., smooth muscle, 
endothelium, collagen, and elastin remodel in response to disease. Nonlinear optical microscopy, available in our department, can facilitate the studies.

Mathematical modeling is another area that can be quickly started based on this study as the experimental data was modeling-friendly. Nearly all of the parameters useful for arteriolar modeling, e.g., biaxial stress-stretch relationships and levels of activation induced by different agonists, have been recorded. Constitutive formulas proposed for microvessels in the literature are based on modifications of formulas for large vessels. As arteriolar wall morphology, composition, and associated passive mechanical behaviors are distinct from conduit arteries, the validity of using material parameters of big vessels for arterioles could be compromised. Our data, on the other hand, will support a better modeling for arterioles.

Arteriolar culture is a big experimental challenge as the arteriole must remain active and disinfected under long-term tests, which is different from this short-term study. Such culture, if successfully executed, will render rich information for mechanobiology of endothelium and smooth muscle, i.e., stress-induced acute response as well as long-term growth and remodeling, which may finally help elucidate certain questions that can not be answered from the current study, e.g., the absence of optimal length and the effect of axial stretch on growth and remodeling. Arteriolar imaging using confocal microscopy or other devices can be coupled with the culture setup to provide 3D luminal morphology. 


\section{REFERENCES}

1. Aukland $\mathbf{K}$ and Oien AH. Renal autoregulation: models combining tubuloglomerular feedback and myogenic response. Am J Physiol 252: F768-783, 1987.

2. Bakker EN, Sorop O, Spaan JA, and VanBavel E. Remodeling of resistance arteries in organoid culture is modulated by pressure and pressure pulsation and depends on vasomotion. Am J Physiol Heart Circ Physiol 286: H2052-2056, 2004.

3. Bakker EN, van der Meulen ET, van den Berg BM, Everts V, Spaan JA, and VanBavel E. Inward remodeling follows chronic vasoconstriction in isolated resistance arteries. J Vasc Res 39: 12-20, 2002.

4. Bakker EN, Versluis JP, Sipkema P, VanTeeffelen JW, Rolf TM, Spaan JA, and VanBavel E. Differential structural adaptation to haemodynamics along single rat cremaster arterioles. J Physiol 548: 549-555, 2003.

5. Baumbach GL. Effects of increased pulse pressure on cerebral arterioles. Hypertension 27: 159-167, 1996.

6. Baumbach GL and Hajdu MA. Mechanics and composition of cerebral arterioles in renal and spontaneously hypertensive rats. Hypertension 21: 816-826, 1993.

7. Baumbach GL, Siems JE, Faraci FM, and Heistad DD. Mechanics and composition of arterioles in brain stem and cerebrum. Am J Physiol 256: H493-501, 1989.

8. Baumbach GL, Siems JE, and Heistad DD. Effects of local reduction in pressure on distensibility and composition of cerebral arterioles. Circ Res 68: 338-351, 1991. 
9. Baumbach GL, Walmsley JG, and Hart MN. Composition and mechanics of cerebral arterioles in hypertensive rats. Am J Pathol 133: 464-471, 1988.

10. Bayliss WM. On the local reactions of the arterial wall to changes of internal pressure. J of Physiol (Lond) 28: 220-231, 1902.

11. Brekke JF, Gokina NI, and Osol G. Vascular smooth muscle cell stress as a determinant of cerebral artery myogenic tone. Am J Physiol Heart Circ Physiol 283: H2210-2216, 2002.

12. Burrowes KS, Hunter PJ, and Tawhai MH. Anatomically based finite element models of the human pulmonary arterial and venous trees including supernumerary vessels. J Appl Physiol 99: 731-738, 2005.

13. Burrows ME and Johnson PC. Arteriolar responses to elevation of venous and arterial pressures in cat mesentery. Am J Physiol 245: H796-807, 1983.

14. Cipolla MJ, Gokina NI, and Osol G. Pressure-induced actin polymerization in vascular smooth muscle as a mechanism underlying myogenic behavior. Faseb J 16: 72$76,2002$.

\section{Clerin V, Nichol JW, Petko M, Myung RJ, Gaynor JW, and Gooch KJ.} Tissue engineering of arteries by directed remodeling of intact arterial segments. Tissue Eng 9: 461-472, 2003.

16. Cox RH. Arterial wall mechanics and composition and the effects of smooth muscle activation. Am J Physiol 229: 807-812, 1975.

17. Cox RH. Comparison of arterial wall mechanics using ring and cylindrical segments. Am J Physiol 244: H298-303, 1983. 
18. Cunningham JJ, Linderman JJ, and Mooney DJ. Externally applied cyclic strain regulates localization of focal contact components in cultured smooth muscle cells. Ann Biomed Eng 30: 927-935, 2002.

19. Davis MJ. Myogenic response gradient in an arteriolar network. Am J Physiol 264: H2168-2179, 1993.

20. Davis MJ and Gore RW. Length-tension relationship of vascular smooth muscle in single arterioles. Am J Physiol 256: H630-640, 1989.

21. Davis MJ and Hill MA. Signaling mechanisms underlying the vascular myogenic response. Physiol Rev 79: 387-423, 1999.

22. Davis MJ, Kuo L, Chilian WM, and Muller JM. Isolated, perfused microvessels. In: Clinically Applied Microcirculation Research, edited by Barker JH, Anderson GL and Menger MD. Boca Raton, FL: CRC Press, 1995.

23. Doubal S, Klemera P, Semecky V, Lamka J, and Kucharova M. Non-linear mechanical behavior of visco-elastic biological structures--measurements and models. Acta Medica (Hradec Kralove) 47: 297-300, 2004.

24. Driessen NJ, Bouten CV, and Baaijens FP. A structural constitutive model for collagenous cardiovascular tissues incorporating the angular fiber distribution. $J$ Biomech Eng 127: 494-503, 2005.

25. Duling BR, Gore RW, Dacey RG, Jr., and Damon DN. Methods for isolation, cannulation, and in vitro study of single microvessels. Am J Physiol 241: H108-116, 1981. 
26. Dunn WR, Wellman GC, and Bevan JA. Enhanced resistance artery sensitivity to agonists under isobaric compared with isometric conditions. Am J Physiol 266: H147$155,1994$.

27. Fung YC. Biomechanics: Mechanical Properties of Living Tissues. New York: Springer, 1993.

28. Fung YC. Biomechanics: Motion, Flow, Stress, and Growth. New York: Springer-Verlag, 1990.

29. Glagov S, Vito R, Giddens DP, and Zarins CK. Micro-architecture and composition of artery walls: relationship to location, diameter and the distribution of mechanical stress. J Hypertens Suppl 10: S101-104, 1992.

30. Gleason RL, Gray SP, Wilson E, and Humphrey JD. A multiaxial computercontrolled organ culture and biomechanical device for mouse carotid arteries. J Biomech Eng 126: 787-795, 2004.

31. Gleason RL and Humphrey JD. Effects of a sustained extension on arterial growth and remodeling: a theoretical study. J Biomech 38: 1255-1261, 2005.

32. Gleason RL, Wilson E, and Humphrey JD. Biaxial biomechanical adaptations of mouse carotid arteries cultured at altered axial extensions. J Biomech (accepted), 2006.

33. Goffin JM, Pittet P, Csucs G, Lussi JW, Meister JJ, and Hinz B. Focal adhesion size controls tension-dependent recruitment of alpha-smooth muscle actin to stress fibers. J Cell Biol 172: 259-268, 2006. 
34. Gore RW and Davis MJ. Mechanics of smooth muscle in isolated single microvessels. Ann Biomed Eng 12: 511-520, 1984.

35. Greensmith JE and Duling BR. Morphology of the constricted arteriolar wall: physiological implications. Am J Physiol 247: H687-698, 1984.

36. Hajdu MA, Heistad DD, Ghoneim S, and Baumbach GL. Effects of antihypertensive treatment on composition of cerebral arterioles. Hypertension 18: II15$21,1991$.

37. Hajdu MA, Heistad DD, Siems JE, and Baumbach GL. Effects of aging on mechanics and composition of cerebral arterioles in rats. Circ Res 66: 1747-1754, 1990.

38. Han HC, Ku DN, and Vito RP. Arterial wall adaptation under elevated longitudinal stretch in organ culture. Ann Biomed Eng 31: 403-411, 2003.

39. Heagerty AM, Aalkjaer C, Bund SJ, Korsgaard N, and Mulvany MJ. Small artery structure in hypertension. Dual processes of remodeling and growth. Hypertension 21: 391-397, 1993.

40. Hill MA and Meininger GA. Calcium entry and myogenic phenomena in skeletal muscle arterioles. Am J Physiol 267: H1085-1092, 1994.

41. Hill MA, Zou H, Davis MJ, Potocnik SJ, and Price S. Transient increases in diameter and $[\mathrm{Ca}(2+)](\mathrm{i})$ are not obligatory for myogenic constriction. Am J Physiol Heart Circ Physiol 278: H345-352, 2000.

42. Hill MA, Zou H, Potocnik SJ, Meininger GA, and Davis MJ. Invited review: arteriolar smooth muscle mechanotransduction: $\mathrm{Ca}(2+)$ signaling pathways underlying myogenic reactivity. J Appl Physiol 91: 973-983, 2001. 
43. Holzapfel GA, Sommer G, Gasser CT, and Regitnig P. Determination of layer-specific mechanical properties of human coronary arteries with nonatherosclerotic intimal thickening and related constitutive modeling. Am J Physiol Heart Circ Physiol 289: H2048-2058, 2005.

44. Humphrey JD. Cardiovascular Solid Mechanics: Cells, Tissues and Organs. New York: Springer, 2002.

45. Humphrey JD and Wilson E. A potential role of smooth muscle tone in early hypertension: a theoretical study. J Biomech 36: 1595-1601, 2003.

46. Intengan HD, Deng LY, Li JS, and Schiffrin EL. Mechanics and composition of human subcutaneous resistance arteries in essential hypertension. Hypertension 33: 569-574, 1999.

47. Izzard AS, Bund SJ, and Heagerty AM. Myogenic tone in mesenteric arteries from spontaneously hypertensive rats. Am J Physiol 270: H1-6, 1996.

48. Jackson PA and Duling BR. Myogenic response and wall mechanics of arterioles. Am J Physiol 257: H1147-1155, 1989.

49. Jackson ZS, Gotlieb AI, and Langille BL. Wall tissue remodeling regulates longitudinal tension in arteries. Circ Res 90: 918-925, 2002.

50. Kaazempur-Mofrad MR, Isasi AG, Younis HF, Chan RC, Hinton DP, Sukhova G, LaMuraglia GM, Lee RT, and Kamm RD. Characterization of the atherosclerotic carotid bifurcation using MRI, finite element modeling, and histology. Ann Biomed Eng 32: 932-946, 2004. 
51. Kuo L, Chilian WM, and Davis MJ. Coronary arteriolar myogenic response is independent of endothelium. Circ Res 66: 860-866, 1990.

52. Kuo L, Chilian WM, and Davis MJ. Interaction of pressure- and flow-induced responses in porcine coronary resistance vessels. Am J Physiol 261: H1706-1715, 1991.

53. Kuo L, Davis MJ, and Chilian WM. Endothelium-dependent, flow-induced dilation of isolated coronary arterioles. Am J Physiol 259: H1063-1070, 1990.

54. Kuo L, Davis MJ, and Chilian WM. Myogenic activity in isolated subepicardial and subendocardial coronary arterioles. Am J Physiol 255: H1558-1562, 1988.

55. Laurant P, Adrian M, and Berthelot A. Effect of age on mechanical properties of rat mesenteric small arteries. Can J Physiol Pharmacol 82: 269-275, 2004.

56. Laurant P, Touyz RM, and Schiffrin EL. Effect of pressurization on mechanical properties of mesenteric small arteries from spontaneously hypertensive rats. J Vasc Res 34: 117-125, 1997.

57. Leung DY, Glagov S, and Mathews MB. Cyclic stretching stimulates synthesis of matrix components by arterial smooth muscle cells in vitro. Science 191: 475-477, 1976.

58. Massett MP, Ungvari Z, Csiszar A, Kaley G, and Koller A. Different roles of PKC and MAP kinases in arteriolar constrictions to pressure and agonists. Am J Physiol Heart Circ Physiol 283: H2282-2287, 2002.

59. McHale NG and Roddie IC. The effect of transmural pressure on pumping activity in isolated bovine lymphatic vessels. J Physiol 261: 255-269, 1976. 
60. Meininger GA and Davis MJ. Cellular mechanisms involved in the vascular myogenic response. Am J Physiol 263: H647-659, 1992.

61. Miller FJ, Jr., Dellsperger KC, and Gutterman DD. Myogenic constriction of human coronary arterioles. Am J Physiol 273: H257-264, 1997.

62. Muller-Delp J, Spier SA, Ramsey MW, Lesniewski LA, Papadopoulos A, Humphrey JD, and Delp MD. Effects of aging on vasoconstrictor and mechanical properties of rat skeletal muscle arterioles. Am J Physiol Heart Circ Physiol 282: H1843-1854, 2002.

63. Mulvany MJ. Small artery remodeling and significance in the development of hypertension. News Physiol Sci 17: 105-109, 2002.

64. Na S, Sun Z, Meininger GA, and Humphrey JD. On atomic force microscopy and the constitutive behavior of living cells. Biomech Model Mechanobiol 3: 75-84, 2004.

65. Neves MF, Virdis A, and Schiffrin EL. Resistance artery mechanics and composition in angiotensin II-infused rats: effects of aldosterone antagonism. $J$ Hypertens 21: 189-198, 2003.

66. Ohta M, Wetzel SG, Dantan P, Bachelet C, Lovblad KO, Yilmaz H, Flaud P, and Rufenacht DA. Rheological changes after stenting of a cerebral aneurysm: a finite element modeling approach. Cardiovasc Intervent Radiol 28: 768-772, 2005.

67. Oien AH and Aukland K. A mathematical analysis of the myogenic hypothesis with special reference to autoregulation of renal blood flow. Circ Res 52: 241-252, 1983. 
68. Osol G, Brekke JF, McElroy-Yaggy K, and Gokina NI. Myogenic tone, reactivity, and forced dilatation: a three-phase model of in vitro arterial myogenic behavior. Am J Physiol Heart Circ Physiol 283: H2260-2267, 2002.

69. Osol G and Halpern W. Myogenic properties of cerebral blood vessels from normotensive and hypertensive rats. Am J Physiol 249: H914-921, 1985.

70. Prewitt RL, Rice DC, and Dobrian AD. Adaptation of resistance arteries to increases in pressure. Microcirculation 9: 295-304, 2002.

71. Raghavan ML, Trivedi S, Nagaraj A, McPherson DD, and Chandran KB. Three-dimensional finite element analysis of residual stress in arteries. Ann Biomed Eng 32: $257-263,2004$.

72. Skalak TC and Price RJ. The role of mechanical stresses in microvascular remodeling. Microcirculation 3: 143-165, 1996.

73. Sun D, Kaley G, and Koller A. Characteristics and origin of myogenic response in isolated gracilis muscle arterioles. Am J Physiol 266: H1177-1183, 1994.

74. Sun D, Messina EJ, Kaley G, and Koller A. Characteristics and origin of myogenic response in isolated mesenteric arterioles. Am J Physiol 263: H1486-1491, 1992.

75. Szekeres M, Nadasy GL, Dezsi L, Orosz M, Tokes A, and Monos E. Segmental differences in geometric, elastic and contractile characteristics of small intramural coronary arteries of the rat. J Vasc Res 35: 332-344, 1998. 
76. Takamizawa $\mathbf{K}$, Hayashi $\mathbf{K}$, and Matsuda $\mathbf{T}$. Isometric biaxial tension of smooth muscle in isolated cylindrical segments of rabbit arteries. Am J Physiol 263: H30-34, 1992.

77. Wilson E, Mai Q, Sudhir K, Weiss RH, and Ives HE. Mechanical strain induces growth of vascular smooth muscle cells via autocrine action of PDGF. $J$ Cell Biol 123: 741-747, 1993.

78. Zou H, Ratz PH, and Hill MA. Role of myosin phosphorylation and $[\mathrm{Ca} 2+] \mathrm{i}$ in myogenic reactivity and arteriolar tone. Am J Physiol 269: H1590-1596, 1995.

79. Zou H, Ratz PH, and Hill MA. Temporal aspects of $\mathrm{Ca}(2+)$ and myosin phosphorylation during myogenic and norepinephrine-induced arteriolar constriction. $J$ Vasc Res 37: 556-567, 2000.

80. Zulliger MA, Kwak NT, Tsapikouni T, and Stergiopulos N. Effects of longitudinal stretch on VSM tone and distensibility of muscular conduit arteries. Am $J$ Physiol Heart Circ Physiol 283: H2599-2605, 2002. 


\section{VITA}

Name: Hong Guo

Address: c/o Dr. Jay Humphrey, Department of Biomedical Engineering, 337 Zachry

Engineering Center, 3120 TAMU, College Station, TX 77843

Email: iamguohong@yahoo.com

Education: M.S., Biomedical Engineering, Texas A\&M University, USA, 2006

B.S., Mechanics and Engineering Science, Fudan University, China, 2002 